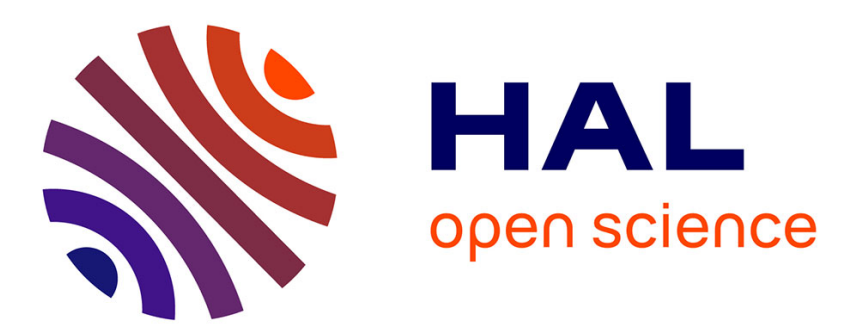

\title{
La confiance : construction, déconstruction, reconstruction du concept; description-évaluation du phénomène
}

Denis Benoit

\section{- To cite this version:}

Denis Benoit. La confiance: construction, déconstruction, reconstruction du concept; description-évaluation du phénomène. Communication et Management, 2018, 15 (2), pp.5-34. 10.3917/comma.152.0005 . hal-02158161v1

\section{HAL Id: hal-02158161 \\ https://hal.science/hal-02158161v1}

Submitted on 17 Jun 2019 (v1), last revised 15 Jul 2019 (v2)

HAL is a multi-disciplinary open access archive for the deposit and dissemination of scientific research documents, whether they are published or not. The documents may come from teaching and research institutions in France or abroad, or from public or private research centers.
L'archive ouverte pluridisciplinaire HAL, est destinée au dépôt et à la diffusion de documents scientifiques de niveau recherche, publiés ou non, émanant des établissements d'enseignement et de recherche français ou étrangers, des laboratoires publics ou privés.

\section{(1) $(1) \Theta$}

Distributed under a Creative Commons Attribution - NonCommercial - NoDerivatives| 4.0 


\title{
La confiance : construction, déconstruction, reconstruction du concept ; description-évaluation du phénomène
}

\author{
Denis BENOIT \\ Professeur (S.I.C.), Université Montpellier 3, I.T.I.C., LERASS-CERIC
}

«Osez renoncer à toutes les phrases, toutes les paroles et tous les mots dont vous n'avez pas l'expérience »

Arnaud Desjardins

"Que l'on arrête de nous tanner en disant que les gens sont naturellement méfiants! A priori, ils se font confiance et ont besoin d'un système où ils peuvent faire confiance »

Matthieu Ricard

Pour le groupe $\operatorname{Org} \& C o$ (SFSIC), Catherine De Lavergne avait organisé, en avril 2015, une journée d'études axée sur un thème qui lui tenait, sans aucun doute, particulièrement à cœur : celui de la confiance, plus particulièrement la confiance en communication. Et elle m'avait demandé à cette occasion de préparer une contribution personnelle.

Le texte ci-dessous, qui en condense le contenu, est dédié à une enseignantechercheure qui a énergiquement contribué à assurer au département InformationCommunication de l'Université Montpellier 3, auquel elle appartenait, le développement qu'on lui connaît aujourd'hui.

\section{La confiance en tant que concept, que mot, qu'objet, que phénomène}

Aborder dans une visée pragmatique et praxéologique le concept, le mot mais aussi l'objet, le phénomène "confiance" dans son entière complexité nous paraît impliquer de façon quasi inéluctable un (compendieux) exposé préliminaire axé sur une tentative d'élucidation de la notion ou du concept de concept ${ }^{1}:$ qu'entend-on au juste - et que peut-on entendre - par le terme « concept » ? Pour ce faire - et pour ne pas multiplier les références à l'ensemble conséquent de travaux (notamment linguistiques et philosophiques) disponibles sur ce thème -, nous nous fonderons sur la réflexion menée par le philosophe et sinologue François Jullien (2014) dans le cadre de sa conférence "Qu'est-ce qu'un concept? $»^{2}$.

\footnotetext{
${ }^{1}$ De façon sommaire, une notion peut être définie comme «une connaissance intuitive, générale qui synthétise les caractères essentiels d'un objet, mais ne prétend pas à la scientificité », (Dictionnaire le Nouveau Petit Robert, 2008); et un concept comme «une représentation mentale générale et abstraite d'un objet [...] stabilisée dans une communauté de savoirs à un moment déterminé (concept de poids en physique, de raison en philosophie, de verbe en grammaire). Aussi, rigoureusement parlant, le terme de concept fait référence à un objet construit dans le monde scientifique ou savant », (Chartrand, De Koninck, 2009). Pour le philosophe Gilles Deleuze (1991), «la philosophie est l'art de former, d'inventer, de fabriquer des concepts ».

${ }^{2}$ In « Cours méthodiques et populaires de philosophie », 12 novembre 2014 - Bibliothèque nationale de France, voir le lien https:/www.youtube.com/watch?v=P116R_V312I (consulté le 26.11.2018).
} 
Pour Jullien, un concept se différencie d'une représentation (simple idée) ou d'une notion (ébauche de connaissance). C'est un « produit », un outil fonctionnel et opératoire, permettant de «saisir» (begrifen, selon le philosophe Emmanuel Kant), élaborant, construisant, la pensée : c'est l'outil de la connaissance (particulièrement philosophique). Le concept configure, unit, totalise un « tout» en marquant des limites, en définissant par exemple : qu'est-ce que le courage ou la confiance, quel est le tout du courage ou de la confiance?, et non pas le courage, la confiance appréhendés dans des cas d'espèce singuliers. Ce faisant, il s'agit de passer de la diversité des possibles contingents à l'unité abstraite, à l'" en soi », à l'absolu (par exemple, du "qu'est-ce qui est beau? ", à «qu'est-ce que le beau? »). Le concept totalise, abstrait mais également « catégorise»: autrement dit, il range, il ordonne, il met en forme, il attribue (prédication). Ainsi, selon Kant, pour connaître je ne dispose que de mes sens (empirisme), mais cette connaissance est informe, diverse, changeante, inconsistante et c'est l'esprit qui la formalise par les concepts que produit l'entendement. La véritable connaissance est donc composée d'une expérience sensorielle associée à l'outil conceptuel (entendement) qui la met en forme : concevoir, c'est «mettre en forme le divers empirique sous l'unité du concept produit ». Pour Kant, l'opération essentielle à exécuter est de mettre en rapport sensible et intelligible par l'entremise d'une fonction (médiatrice) : l'imagination, soit l'interface entre sensible et intelligible, en prise sur le divers de la perception sensible et relayée par l'entendement, qui produit l'unité synthétique.

Ainsi défini, le concept apparaît toutefois comme une forme statique, immobile, rigide, manquant le mouvement de la vie. Cependant, en référence à Georg Wilhelm Friedrich Hegel, l'on peut dire que, de facto, l' « en soi » est travaillé par l'opposition, le négatif (le «non ») ; opposé, contradiction qui n'est alors pas «en dehors », mais «en dedans» : c'est «La Vie» qui cherche à s'introduire dans l'unité rigide du concept. Ainsi, si le concept totalise, abstrait, catégorise, il doit (devrait) également " dialectiser », et la contradiction être portée en son sein même (l'autre réside dans le même). Car le contraire n'est pas extérieur à l'essence, il est déjà au travail en elle-même: toute détermination est liée à une négation au sein du soi, du même, ce qui entrâne un autodéveloppement, un autodépassement, un devenir dynamique, dialectique. Ici, il convient de travailler contre le langage, qui définit des qualités, des propriétés, des «en $s o i »$, en bref, qui reste statique en définissant, et la pensée dialectique se doit de lutter contre les identifications auxquelles aboutissent les définitions qu'il pose: il s'agit toujours d'introduire de l'autre dans le même et, par exemple, penser ce qu'il y a de faiblesse dans la force et inversement. Qu'est-ce qui échappe au concept considéré en tant que saisie, que prise ? En fait, le concept ne peut que très difficilement « s'échapper » du mot qui le désigne (par exemple, l'effort du concept pour s'abstraire du terme « concept» apparaît conséquent...). Le concept doit saisir mais également " enfanter »-selon Hegel, le concept est le gland qui donne l'arbre (et il n'est alors plus le begriff allemand mais plutôt le concetto italien). Dès lors, le concept est-il universel ? Existe-t-il des concepts suffisamment abstraits pour s'extraire du divers, des cultures ? Jusqu'où l'abstraction par rapport à la langue qui porte le concept est-elle envisageable ? Les catégories kantiennes - les « concepts souches » de l'entendement : unité, pluralité, totalité, négation, etc. sont-elles universelles ? Pour Jullien, il semble bien qu'il faille répondre par la négative à ce type de questions: les catégories sont toujours celles d'une langue particulière, singulière. Par exemple, la «substance », c'est ce qui se tient sous dans un contexte de 
langue européeenne mais, dans le contexte de la langue chinoise, la substance disparaît (l'opposition existence / non existence, notamment, n'est pas marquée en chinois qui pense l'«entre-existence », le flou, l'indistinct). De fait, l'abstraction n'est jamais aboutie, l'on n'a jamais complètement abstrait la pensée de la langue. Certes le concept unifie le divers mais cette unité, cette identité produite n'est-elle pas factice ? Pour Friedrich Nietzsche, les concepts permettent de nous répérer dans la diversité de la vie, de nous aider à vivre, mais ce sont de simples étiquettes unitaires de rangement commodes, des «fictions conventionnelles qui servent à désigner, à se mettre d'accord, nullement expliquer quoique ce soit »: abus, fiction des concepts qui rangent le divers sous une unité de facade, (ce sont au mieux des "signes de reconnaissance», et non pas de la connaissance stricto sensu).

Jullien conclut sa conférence en affirmant pourtant qu'il s'agit, in fine, de critiquer une telle critique : un concept est un " outil possible de la pensée », remplissant certaines fonctions, impliquant certains usages, qui exécute des opérations déterminées; la conceptualisation n'est pas illusion, mais remplit seulement certaines fonctions, spécifiques, et non d'autres ${ }^{3}$.

Ainsi, un concept apparaît comme la traduction en (un) mot(s) d'une représentation (pensée) que du reste, concomitamment (ou récursivement), le mot (contribue à) structure(r) ; mot (et concept), qui, par définition, manque(nt) toujours à la fois la parfaite transcription de la pensée (conceptuelle, conceptualisante) et le « réel » qu'il(s) vise(nt) à déterminer.

De fait, comme la carte n'est pas le territoire (Korzybski, 1933, passim), les mots, «corporification des idées » (Lordat, cité in Laplane, 1999, passim), se distinguent des choses qu'ils nomment. Et le discours, par suite de cette différence de nature, ne peut jamais, quelque soit sa précision et sa prolixité, parfaitement, définitivement cerner, saisir la véritable, l'ontologique réalité desdites choses ${ }^{4}$.

Dès lors, une question connexe apparaît : existe-t-il (véritablement) des concepts hors les mots, le discours, le langage ? Peut-on (véritablement) penser (concevoir, conceptualiser) sans langage ; pensée «non verbale » qui serait mieux performante pour investir la réalité des choses telle qu'elle est? Tout dépend évidemment comment l'on définit le terme «concept » et ce que l'on nomme «pensée »; en bref, tout dépend de la construction réalisée des concepts de concept et de pensée...

Ainsi, pour un nombre (aujourd'hui) croissant d'auteurs, les processus que l'on regroupe communément sous le terme de "pensée » peuvent être non seulement de type verbal mais aussi (notamment) de type visuel : par exemple, deux auteurs (Cooper, Shepard, 1985, pp. 40-47) considéraient-ils dès les années 80 (sur la base d'expériences menées sur des singes) que : «certaines pensées sont verbales, comme si nous parlions

\footnotetext{
${ }^{3}$ L'on peut alors considérer les concepts non pas tant comme des descriptions du réel tel qu'il est, mais comme des descriptions des processus de connaissance adoptés un temps par les chercheurs, et susceptibles, à terme, d'évoluer sinon de changer du tout au tout, cf. la notion de « paradigme », chère à l'historien des sciences Thomas Kuhn (1962). Descriptions à appréhender en fonction leur utilité, en tout cas si l'on adopte une position constructiviste et, disons, pragmatiste qui met bien plus l'accent sur les effets en aval de ces descriptions que sur leur valeur de vérité, leur véridicité (voir Benoit, 2004, pp. 184-202).

${ }^{4}$ A supposer leur existence « en soi » (cf. notre réflexion relative aux « idéologies » matérialiste/réaliste vs. constructiviste, in Benoit, ibid.). Ainsi, plutôt que de "choses » ou d' "objets », sans doute convient-il, pour simplifier la problématique et le propos, de parler de «phénomènes », c'est-à-dire d' «étants »qui n'existent pas comme réalités indépendantes du processus cognitif qui les connaît.
} 
silencieusement à quelqu'un; d'autres processus mentaux sont visuels : nous modifions les images mentales sans l'aide de mots». Généralisant le propos, le neurologue Dominique Laplane (2001, pp. 345-357) - se fondant sur les recherches menées sur les hémisphères cérébraux et l'asymétrie fonctionnelle, pour lesquelles le neurophysiologiste Roger Wolcott Sperry obtint le prix Nobel - affirmera qu' "une pensée ne peut être complète sans l'intervention du langage, mais [...] elle existe largement préformée sur un mode non verbal [...] Le langage apparaît [...] essentiellement comme une mise en forme de ce qui est déjà apparu à la conscience et qui demande seulement d'être traduit en un code symbolique pour pouvoir être communiqué».

In fine, il semble bien que le concept que nous élaborons en pensée et/ou par le langage, et le mot que nous utilisons pour " corporifier », " incarner » le concept et/ou le construire et le traduire, ne peuvent - du fait de l'(évidente) imperfection inhérente aux processus de conception, de construction et de traduction - que manquer la cible visée, à savoir la chose (ou le phénomène) dont nous tentons de traiter.

En l'occurrence, pour ce qui nous occupe ici, qu'est-ce que la confiance en deçà et au delà du concept et du mot ? Autrement dit, pouvons-nous (paradoxalement) penser et expliquer (par des concepts et par des mots) ce qui - soit la chose et/ou le phénomène « confiance » - à la fois excède (toujours) le concept et ne peut (jamais) s'enfermer dans un simple mot? Pour tenter de résoudre cet apparent paradoxe, nous ferons appel à la fois à la réflexion d'un philosophe et à celle d'un théologien : Alexandre Jollien et Jean-Yves Leloup.

Le premier cite régulièrement dans ses ouvrages ${ }^{5}$ l'un des grands textes du bouddhisme mahāyāna, le Sūtra du diamant ${ }^{6}$, dans lequel un paradoxe est à l'œuvre («le bouddha n'est pas le bouddha, c'est pourquoi je l'appelle le bouddha»), et il explique : «Une lecture m'habite, me déroute et me convertit sans cesse. Je viens de lire le Soûtra du Diamant. Un refrain revient sans cesse, une logique paradoxale jalonne le discours du Bouddha: 'X n'est pas X, par conséquent, je l'appelle X.' Rarement, énoncé $m$ 'a autant aidé. Je l'emploie partout et toujours dans mon quotidien, enfin j'essaie. 'Ma femme n'est pas ma femme c'est pourquoi je l'appelle ma femme.', 'mes enfants ne sont pas mes enfants, c'est pourquoi je les appelle mes enfants.' Le Bouddha invite à dégommer toutes les étiquettes. Ma femme n'est pas ma femme. Elle est beaucoup plus riche, beaucoup plus dense, beaucoup plus unique, beaucoup plus insolite que ce que j'en perçois. Et ainsi en va-t-il pour mes enfants, pour mes amis, pour la réalité, bref, pour le monde. Nos étiquettes figent le réel, le rétrécissent, le tuent. Mais lutter contre les étiquettes est encore une posture, une fixation. Le Tathagata [l'une des épithètes désignant le Bouddha] invite à aller plus loin. Le cour libre peut utiliser les étiquettes et appeler un chat un chat. Du moment que je sais que ma femme n'est pas ma femme et que jamais je ne pourrai la saisir dans des concepts, je peux librement, avec légèreté, l'appeler ma femme. Le malheur, c'est de se fixer dans les étiquettes, se figer dans ce qu'on a été et dans ce qu'on est. Ainsi, aujourd'hui, je me suis dit 'Alexandre n'est pas Alexandre. L'Alexandre d'hier n'est déjà plus. Celui qui est fatigué en ce moment mourra dans la journée pour naître nouveau.' La non fixation, c'est peut-être de laisser mourir ce moi

\footnotetext{
${ }^{5}$ Voir par exemple Petit traité de l'abandon, 2012, passim.

${ }^{6}$ Pour le texte lui-même, voir par exemple le lien http://mpcmontreal.org/?q=fr/node/551 (consulté le 27.05.2016).
} 
fatigué, humilié, content parfois, gratifié et heureux souvent. La non fixation, c'est se laisser vivre plutôt que vivre $»^{7}$.

De fait, pour Jollien, concepts et mots « enferment les choses et les gens » - c'est seulement lorsque j'en suis conscient que je peux en faire un usage non réducteur, non simpliste : la confiance n'est pas la confiance, c'est pourquoi je la baptise confiance... -, et il convient de les dépasser : sans pour autant le nier, ne pas définitivement fixer, figer dans des définitions ce que l'on croit être «le réel des objets et des phénomènes »- en l'occurrence celui de la confiance -, mais laisser ce réel se révéler, se déployer, le « laisser être » dans un processus infiniment complexe, celui de « La Vie ».

Le second, le théologien Jean-Yves Leloup, traitant (2014, passim) du « regard» porté par les hommes sur Dieu, avance d'une part que, selon une étymologie possible du terme, l'athée - du grec ancien $\alpha \theta \varepsilon o \varsigma$, atheos (« sans Dieu»), et de thea « la vue »-, c'est celui qui, " malade du regard », est incapable de voir ce qu'il a devant les yeux, soit l'invisible: "l'athée c'est celui qui voit le visible sans l'invisible qui l'enveloppe, celui qui ne voit pas le jour [...] voir Dieu [du latin dies, "le jour »] c'est voir le jour et le jour ça ne se voit pas, [mais] c'est ce qui nous permet de voir toutes choses. Donc, un athée dans le sens premier du terme c'est quelqu'un [...] qui voit les mille et une choses qui apparaissent dans le jour mais ne perçoit pas cette clarté qui est au cœur des choses, autour des choses et qui est peut-être l'essentiel $\|^{8}$. D'autre part, Leloup (ibid.) considère que "prétendre connaître Dieu c'est une forme d'idolâtrie [...] l'on prend nos idées sur Dieu, nos pensées sur Dieu, nos représentations de Dieu, nos expériences de Dieu pour Dieu lui-même [...] c'est aussi une maladie des yeux». Et, de fait, pour lui, "[si] l'athéisme est une maladie des yeux, la religion aussi ».

Or, si l'on substitue les termes d' «essence» ou d' " être » au mot «Dieu »essence ou être "suprême »-, l'on saisit alors que l'être, l'essence d'un objet quel qu'il soit, restent «toujours au delà de ce que l'on peut [en penser et] en dire ». Par suite, le « réel» de toutes choses est inaccessible par l'intermédiaire des mots et des concepts : ceux que l'on forme pour en cerner la substance manquent toujours ce qu'ils visent.

L'on comprend que Leloup rejoint Jollien et que, pour lui, le concept et le mot «confiance», dans l'optique qui nous intéresse ici - ne peuvent que faillir à saisir l'essence, la substance de la «chose» (confiance) : "le réel, on ne le connait qu'à travers les réalités [...] mais son essence nous échappe toujours, elle est toujours en deçà, au delà, mais on peut idolâtrer la forme ». En conséquence, il s'agit (pour lui) de passer - y compris par une ascèse utilisant paradoxe, méditation, kôan ${ }^{9}$, etc. - au delà du « mental », de l' « intellect », de l'« ego » ${ }^{10}$, au delà des représentations toujours bornées, pour accéder à des «états de conscience», des «niveaux de réalité » qui ne "chosifient», ne réifient pas le réel mais le laissent plutôt se manifester dans son inépuisable densité.

Mettant en œuvre l'attitude proposée par ces deux chercheurs - qui consiste, pour une bonne part, à assumer la contradiction ou le paradoxe résidant dans le fait d'affirmer

\footnotetext{
${ }^{7}$ In «Pharmacopée $\mathrm{n}^{\circ} 18$ » : voir le lien http://www.alexandre-jollien.ch/?p=1020 (consulté le 27.05.2016).

${ }^{8}$ In « Deux maladies des yeux : athéisme et religion », France Culture, « Les racines du ciel », émission du 10/05/2015, sur le lien http://www.franceculture.fr/emissions/les-racines-du-ciel/les-deux-maladies-desyeux-atheisme-et-religion-avec-jean-yves-le-loup (consulté le 28.05.2016).

${ }^{9}$ Voir notamment l'ouvrage de Taïkan Jyoji, L'Art du kôan zen (2001, passim).

10 Selon la signification que donnent de ces termes un certain nombre de courants spirituels: voir notamment André Comte-Sponville, Dictionnaire philosophique, 2013, passim.
} 
que mots et concepts ne permettent pas d'accéder au réel d'un objet quel qu'il soit ...tout en les utilisant pour tenter de le faire -, comment appréhender au mieux ce que l'on nomme communément « confiance »?

\section{Confiance relative et confiance absolue}

Pour le sens commun, la confiance apparait comme une notion dont l'acception est à la fois simple et extrêmement complexe, aujourd'hui particulièrement analysée et usitée quelque soit le domaine - et particulièrement celui des sciences humaines et sociales : disons, pour aller vite, de la Philosophie à l'Économie et au Marketing sous toutes leurs formes. En fait, "il existe tant de définitions de la confiance qu'une seule définition serait insuffisante pour capturer l'essence du concept » (Gagné, 2011, passim). Dès lors, comme avec l'utilisation d'un entonnoir, partons de haut et visons large pour préciser peu à peu nos idées et notre propos sur le thème. Et puisqu'il est parfois dit, avec perspicacité nous semble-t-il, que celui qui possède la véritable sagesse, c'est le naïf, sinon l'idiot - celui qui n'a pas fait d'études, mais qui en remontre aux intellectuels de métier $^{11}$-, d'emblée, faisons intervenir, sages parmi les sages, un poète dont les propos seront soutenus et développés par ceux d'un psychothérapeute de métier. Puis, pour suivre, aussi vrai qu' «une image vaut mille mots» (sentence attribué à Confucius), complétons cette intervention par l'interprétation de l'extrait d'un dessin animé : certains de ses personnages pourront (peut-être) nous en apprendre instantanément beaucoup plus sur la confiance que nous ne pourrions le faire en compulsant un sinon plusieurs ouvrages complexes dédiés au sujet...

Le poète, c'est Christian Bobin qui, interrogé sur sa définition de la confiance ${ }^{12}$, assure que : "le soubassement de cette vie [...] c'est la confiance. Je ne saurais dire en quoi ou en qui. Mais peut-être [est-ce] plus prudent de ne pas dire en quoi ou en qui, de ne pas fermer avec des définitions ou des mots [...] C'est une sorte de tranquillité un peu étrange [...] Le fond même fracassé de la vie délivre une certaine douceur [...] parfois. Mais si vous apercevez cette douceur [...] une fois, c'est pour toujours [...]. Une fois suffit [...] c'est dans l'expérience et donc vous la portez tout le temps [...] vous l'avez vue ne serait-ce qu'une seconde et cette seconde échappe au temps».

On comprend bien que Bobin parle ici d'une sorte d' "absolu de la confiance», une confiance dans le monde, le réel et son infinité d'objets, en bref, dans « La Vie ». Et que ce n'est pas la confiance en l'autre, la confiance - on pourrait peut-être dire 《 relationnelle » et/ou « communicationnelle »-qu'il évoque et qu'il cible, à savoir celle qui relie les hommes entre eux.

Toutefois, c'est le sociologue Louis Quéré (2001, passim) qui affirme qu' : «il n'est pas sûr qu'il y ait un sens à parler de confiance lorsqu'il s'agit de se fier à la stabilité de l'environnement ou à la régularité de comportement de ses objets. En effet, le cas paradigmatique de la confiance est celui d'une relation de confiance entre deux personnes. Les traits caractéristiques de la confiance se maintiennent-ils hors de ce

\footnotetext{
${ }^{11} \mathrm{Cf}$. le lien http://www.academia.edu/9085952/Philosopher cest faire lidiot (consulté le 17.06.2016).

${ }^{12}$ Cf. l'émission de France Culture La confiance, « Les racines du ciel », décembre 2013 (rediffusion), voir le lien http://www.franceculture.fr/emissions/les-racines-du-ciel/rediffusion-la-confiance-avec-christianbobin, (consulté le 17.06.2016) dans laquelle le poète est interviewé à l'occasion de la publication de son ouvrage L'homme-joie (2012, passim).
} 
contexte? Il est possible que nous commettions un abus de langage lorsque nous parlons de faire confiance à un objet ou à une institution ».

Pourtant, a contrario de ce point de vue porté par Quéré, peut-être peut-on dire que nous avons besoin, en nous-mêmes, de cette sorte, d' «absolu de la confiance », de cette confiance « dans le réel et ses objets », pour pouvoir, au quotidien, extraire de cette forme - en fait de ce qui pourrait bien constituer une sorte de " réservoir »-, en puiser, disons, des attitudes, des comportements de type confiant, et les diffuser dans tout un ensemble de cas singuliers, notamment en rapport avec nos relations humaines.

C'est ainsi que le psychiatre et psychothérapeute Christophe Massin débute son ouvrage publié sur le thème de la confiance (2016, passim) par un essai d'élucidation de ce qu'il nomme "peur psychologique », et il y pose la question de savoir comment se dégager de l'emprise de cette dernière, afin de « trouver le sol ferme de la confiance ${ }^{13}$; confiance qu'il fractionne en confiance « relative » et « absolue ».

En fait, Massin commence par distinguer les peurs « animales », qui surgissent au contact d'événements dangereux - utiles et même nécessaires, elles permettent in fine de protéger son intégrité (notamment) physique -, des peurs " psychologiques » mémorisées pendant l'enfance, lors de situations subjectivement vécues comme dangereuses ${ }^{14}$; dernières peurs dont l'enfant extrait des «lois » inconscientes productrices de méfiance, de comportements d'évitement, etc., qui vont trouver à s'appliquer, à se «projeter» de façon quasi automatique, non pertinente, disproportionnée, dans les conjonctures les plus variées rencontrées tout au long de la vie. Autrement dit, des peurs latentes - du rejet, de l'abandon, par exemple - ressurgissent du passé, se manifestent de façon incongrue dans tous les domaines de la vie et conditionnent et perturbent l'adulte qui, ne les reconnaissant pas en tant que telles, attribue à d'autres causes son anxiété, ses déboires et ses échecs, en bref, son incapacité à atteindre une certaine forme d'équanimité sinon de sérénité malgré, bien évidemment, les inéluctables difficultés, épreuves, bouleversements inhérents à l'existence - qu'il ne convient certes pas, lorsqu'ils sont concrets et effectifs, de dénier ou de refuser.

Dans une optique thérapeutique, un premier cheminement consiste, pour Massin, à mettre en évidence cette ou ces peurs se situant en «arrière-plan ${ }^{15}$, à les reconnaitre en tant que telles, à les ressentir dans son corps (et non uniquement à les penser, les analyser «intellectuellement»), à les exprimer, à oser les affronter (pas à pas, avec bienveillance envers soi-même et dans un esprit d'exploration, d'aventure). Il s'agit de parvenir (peu à peu) à quitter le plan imaginaire, fictif qu'elles induisent pour l'« ici et maintenant $»$ de la situation. Hic et nunc qui ancre le sujet dans la réalité actuelle,

\footnotetext{
13 Cf. sur le lien http://www.franceculture.fr/emissions/les-racines-du-ciel/entrer-dans-la-confiance (consulté le 12.06.2016), l'émission Entrer dans la confiance, « Les racines du ciel », France-Culture, le 5.06.2016 (59 min.), et sur le lien https://www.franceinter.fr/emissions/la-tete-au-carre/la-tete-au-carre-11mai-2016 (consulté le 12.06.2016), l'émission Dépasser les peurs et retrouver la confiance, « La tête au carré », France-Inter, le 11.05.2016 (59 min.) ; émissions dans le cadre desquelles est interviewé C. Massin. ${ }^{14}$ Plus fondamentalement, la peur se manifeste pour lui à chaque fois que l'individu se dissocie du monde, ne fait plus «un» avec celui-ci; à savoir lorsque, l' "ego», le «moi » (qui se structure pendant l'enfance) entre en jeu : " Là où il y a deux, il y a la peur » affirme, dans les Upanishads, la philosophie indienne du Vedānta, qui considère l'ego comme " tissé de peur(s) », voir Massin, 2016, pp. 23-26.

${ }^{15}$ Peurs souvent (paradoxalement) « autoréalisatrices » : la peur insidieuse du rejet, par exemple, fait que le sujet à tendance à, de lui-même, se mettre «à part» et, en conséquence, à se sentir rejeté (et parfois sinon souvent à l'être effectivement)...
} 
tangible, celle dans laquelle il peut se mesurer à ces peurs archaïques tout en constatant qu'elles ne l'anéantissent pas et, qu'images mentales, représentations sans support véritablement consistant, fantasmagories évanescentes, elles finissent par s'épuiser d'elles-mêmes, par se déconstruire naturellement.

L'on peut alors entrer dans la confiance, soit ce que Massin définit (à l'instar de Bobin) comme un « abandon » tranquille et lucide en adéquation avec « ce qui est », avec la vérité des faits, l'accueil « réaliste » mais « décrispé », relâché, détendu, fluide, de ce qui vient (lorsque l'élaborer ou le modifier est irréalisable, telle une maladie incurable). Ainsi, la peur affirme "tu ne peux rien», elle fascine et paralyse. Quant à elle, la confiance, de facto liée à un contact consistant, un appui solide, un «sol», un « socle » ferme - la réalité -, si elle ne rejette pas la peur, ne la traite pas en ennemie et autorise sa présence naturelle (clairement identifiée), permet cependant, en toutes situations et circonstances, d'inlassablement poser la question « qu'est-ce que je peux ? ». Immergé dans un réel toujours «sans garantie », c'est-à-dire à jamais définitivement rebelle au contrôle du « moi », apte à constamment priver l' « ego » de ce qu'il veut ardemment, ou générer ce qu'il refuse obstinément, chercher à entrer en contact, à s'ouvrir (de façon aussi posée, paisible mais clairvoyante que possible) à la vie, à l'accepter - y compris dans les troubles, les épreuves, les effrois qu'elle engendre -, permet en retour, dans un cercle vertueux, une causalité circulaire féconde, un accès juste, sagace, et par suite fructueux, au monde « tel qu'il est ».

Par rapport à la confiance " relative », celle que l'ego accorde (ou refuse) à ses propres capacités limitées, la confiance « absolue » serait alors l'intelligence du « Soi », (c'est-à-dire « la nature essentielle de l'être humain », se situant en deçà et au delà de l'ego) ; perspicacité qui, par nature, disposerait de la faculté de répondre pertinemment à toutes situations, quelles qu'elles puissent être. De fait (voir Massin, 2016, pp. 90-91, 135-136, 167-168), il y aurait une confiance relative « ancrée dans le concret », ajustée à la dualité entre le moi et le reste du monde - visant une mise à l'unisson du moi et du monde - pour, dans les différents domaines de notre vie, " agir avec, harmonieusement ». Celle-ci demande des ajustements continuels et "s'attache à reconnaître la gamme particulière des peurs [...] [portée par un sujet] pour les apprivoiser et les traverser». Alors que la confiance dite absolue "se situe sur un autre plan échappant à ces variations, celui de la non-dualité où la distinction sujet/objet disparaît. Immobile et intemporelle, elle n'est pas soumise à des fluctuations »; inconditionnelle, elle est «non dépendante des circonstances, plaisantes ou déplaisantes »: il s'agit d'admettre toutes les contraintes de l'existence, d'accepter tout ce dont nous dépendons pour, justement, devenir non dépendant, libre par rapport à ces contraintes, de "faire confiance à la confiance », de lâcher prise "non comme une défaite mais par simple humilité devant la vérité : accepter la limite, c'est la transcender ».

Pour ce qui concerne maintenant les personnages de dessin animé, il s'agit de Mowgli et du serpent Kaa : l'on découvre dans le film Le Livre De La Jungle ${ }^{16}$ le serpent Kaa face à Mowgli, et l'on entend son célèbre « Aie Confiance »...

\footnotetext{
${ }^{16}$ Film des Studios Disney (1967), inspiré du recueil de nouvelles éponyme de Rudyard Kipling, paru en 1894 ; voir le lien https://www.youtube.com/watch?v=2gflQEF1WQU (consulté le 22.06.2016).
} 


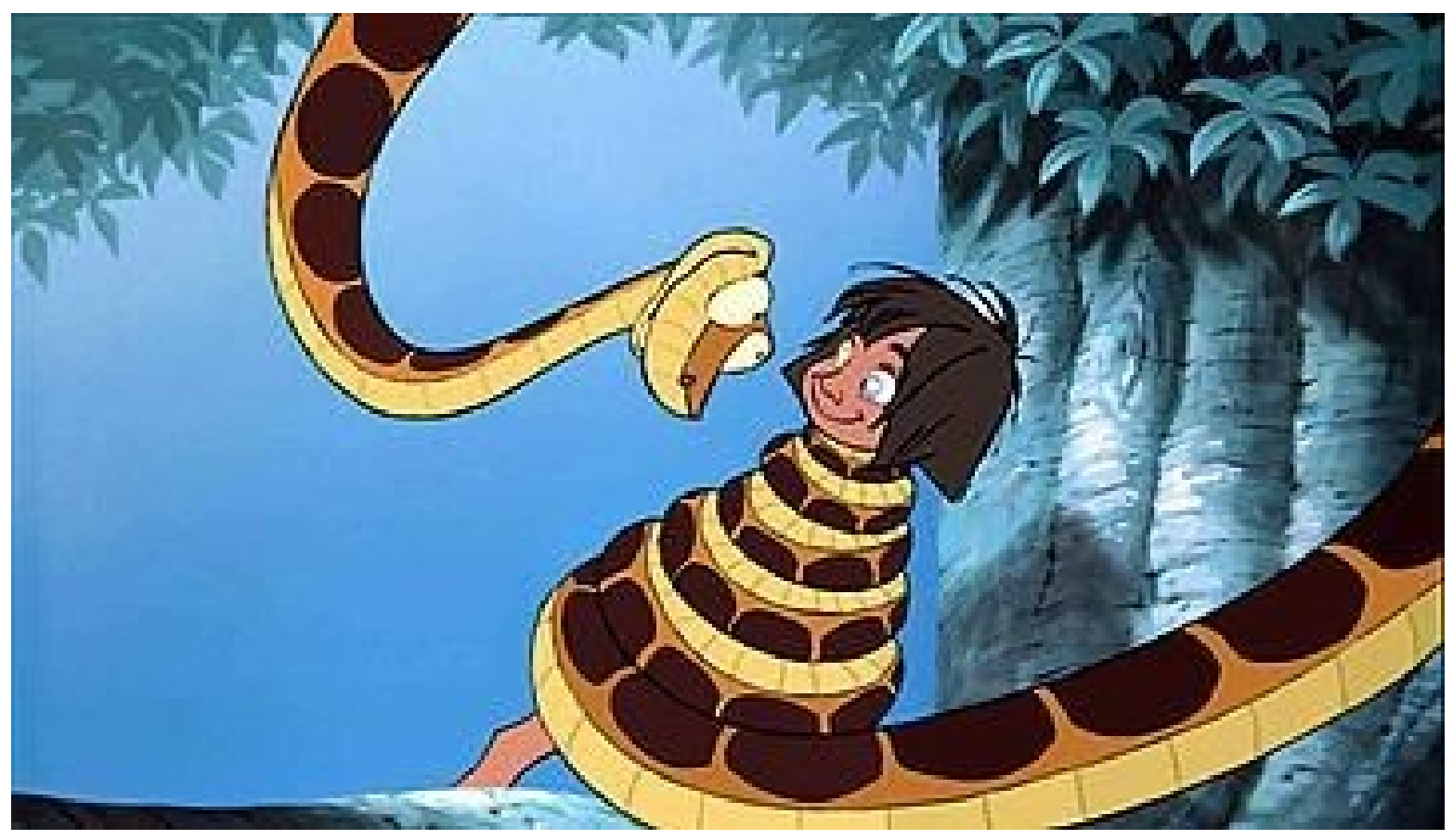

En fait, cet extrait nous paraît poser de façon particulièrement pertinente, sous la forme d'une question, la problématique de la confiance, tout au moins sa partie relative à la relation, à la communication : comment la susciter chez autrui ? En l'occurrence, malgré l'injonction "Aie confiance!", est-ce de la confiance à proprement parler que Kaa cherche à induire chez Mowgli ? Nous ne le pensons pas : ce qu'il recherche, ce serait plutôt à ensorceler, à envoûter Mowgli selon un schéma séduction-persuasion débouchant sur l'extase-fascination de la cible pour, in fine, pouvoir la gouverner et la soumettre, en l'occurrence en faire sa chose et son repas.

Dès lors, il y a bien erreur sur la définition que Kaa propose de son objectif, à savoir que Mowgli ait confiance en lui... Car ce n'est que dans un tout premier temps que Kaa cherche à générer ce que l'on pourrait (éventuellement) baptiser confiance chez Mowgli : seulement pour que ce dernier accepte de croiser son regard narcotique. Après, et très vite, c'est sans doute de tout autre chose dont il s'agit...

Sur de telles bases ainsi énoncées, se posent fatalement des questions du type : qu'est-ce que la confiance " au quotidien », quel est son rôle dans les relations humaines (ou animales), quels sont ses processus d'établissement et de fonctionnement, quels sont ses rapports avec ce que l'on nomme « la communication»?

\section{Confiance au quotidien}

Pour répondre avec pertinence à de telles questions, un sérieux écueil nous paraît devoir être contourné : le fait que certains concepts, que certains mots, possèdent une faculté d'attractivité, un pouvoir de séduction, une aptitude à fasciner considérables comme le serpent Kaa en quelque sorte... - et que, si l'on n'y prend pas garde, l'on se retrouve à essayer de comprendre et d'expliquer exclusivement grâce à eux l'intégralité de la chose ou de la situation que l'on cherche à élucider. 
Ainsi, le concept de confiance est probablement capital pour saisir quelques-uns des processus décisifs du «fonctionnement» humain, de ceux de la communication interpersonnelle et sociale, mais il est sans aucun doute très insuffisant pour les cerner intégralement et, de notre point de vue, doit être utilisé en association étroite avec d'autres notions ou concepts cruciaux : par exemple, ceux de relation, de représentation, d'imitation, d'influence, d'identité, d'engagement, etc. Assemblage subtil entre idées connexes qui, au bout du compte, fera que l'on ne saura plus vraiment reconnaître l'essentiel de l'auxiliaire, de l'accessoire, différencier ce qui est cause et ce qui est effet. Selon nous, une telle façon de procéder peut, dans l'ordre de l'explication, permettre d'obtenir une véritable synergie, certes complexe à mettre en œuvre, mais bien plus efficiente que si l'on s'était limité à considérer indépendamment, isolément, un unique facteur, un unique paramètre, un unique concept explicatif, regardé comme primordial.

Pour permettre de bien saisir le mécanisme que nous cherchons à mettre en évidence, prenons un seul cas d'espèce : la confiance en matière de performance sportive, généralement définie comme "la croyance, la conviction que l'on est capable d'accomplir [en matière de sport] une certaine tâche, ou d'atteindre un certain objectif $\gg$. Sans confiance en soi, il est quasi unanimement reconnu que le sportif ne peut réussir dans son activité - par exemple, descendre sous la barre des quatre minutes pour courir un mile, ce que Roger Bannister a réalisé en 1954, alors que les spécialistes de l'époque s'accordaient tous à dire que le corps humain n'était pas prévu pour cela ...mais Bannister y croyait : il avait confiance en ses capacités et possibilités. Or, la plupart des "coachs » sportifs relient à la notion de confiance celles - notamment - de motivation, d'énergie, d'estime de soi, etc. Mais alors, est-ce la confiance qui est préalable ou initiale en matière de performance sportive ou bien est-ce la motivation, l'énergie ou l'estime de soi (notamment) qui vont la susciter? L'estime de soi, par exemple, est-elle une composante parmi d'autres de la confiance, ou bien est-ce l'inverse ? Or, nous pensons que l'on ne peut répondre à ce type de question et que, dès lors, il convient d'appliquer l'idée d'une causalité circulaire: les causes entrainent des effets qui vont eux-mêmes constituer les causes d'autres effets, et ceci ad infinitum.

En conclusion, affirmons que l'on ne peut se contenter d'un unique concept explicatif qui serait «premier» ou «essentiel»- comme l'indique, du reste, Quéré (ibid., passim) -, mais que les concepts doivent être envisagés en étroite liaison, en connexion rhizomique: pour se référer à Deleuze et Guattari (1980, passim), c'est le modèle descriptif du rhizome, de la structure rhizomatique ou rhizomique qui est probablement à privilégier ; à savoir un modèle dans lequel l'organisation des éléments ne suit pas une ligne de subordination hiérarchique et où tout élément peut en affecter ou en influencer tout autre.

Toutefois, et pour mémoire, précisons qu'un tel modèle doit sans doute être utilisé en association avec le principe dit du « rasoir d'Ockham », formulé dès le XIVème siècle et selon lequel il ne faut pas multiplier les entités sans nécessité ${ }^{17}$. C'est-à-dire que lorsque l'on dispose de plusieurs thèses en compétition qui permettent de prédire exactement les mêmes choses, et que l'on ne peut les départager, la plus simple est la meilleure jusqu'à preuve du contraire : la pluralité de notions ou de concepts ne devrait donc pas être posée sans nécessité, explique ainsi Guillaume d'Ockham.

\footnotetext{
17 Voir par exemple le lien http://www.college-de-france.fr/media/stanislasdehaene/UPL6421126217923963565_R1112_Dehaene.pdf (consulté le 02.07.2016).
} 


\subsection{Signification et définitions communes du terme confiance}

Du latin con- (« ensemble ») et fidere (« se fier », « croire »), la confiance est liée à la foi. Or, étymologiquement, le terme de foi provient du latin fides et se rattache à une racine indo-européenne bheidh, « avoir confiance ».

D'emblée, affirmons qu'il y a sans doute un paradoxe avec la notion de confiance, qui renvoie à la fois à la foi, c'est-à-dire à des croyances irrationnelles (avoir foi en...) mais également à des éléments tout à fait critiques, rationnels et logiques. De fait, l'on sait - au moins intuitivement, cf. les aphorismes «La confiance ne se réclame pas, elle se gagne » (attribué à Marc Goldstein) et «La confiance se gagne en gouttes et se perd en litres » (attribué à Jean-Paul Sartre) -, qu'elle «se gagne » grâce à des attitudes, des manières d'agir, autrement dit qu'elle peut être, et qu'elle est le plus souvent, "non aveugle », à l'inverse de la " foi aveugle», celle que proposait Jésus à Saint Thomas (« Heureux ceux qui croient sans voir! »). Mais qu'au contraire, elle est favorisée et soutenue (ou non) par la répétition, la redondance de certains comportements adéquats.

Pour suivre une telle assertion, reprenons une définition assez largement acceptée ${ }^{18}$ : la confiance est « un état psychologique [...] [fondé sur des] croyances optimistes sur les intentions (ou le comportement) d'autrui ». Et proposons immédiatement une tentative valant ce qu'elle vaut - de champ lexical (soit un groupe de mots se rapportant à une même idée) pour le terme « confiance ${ }^{19}$.

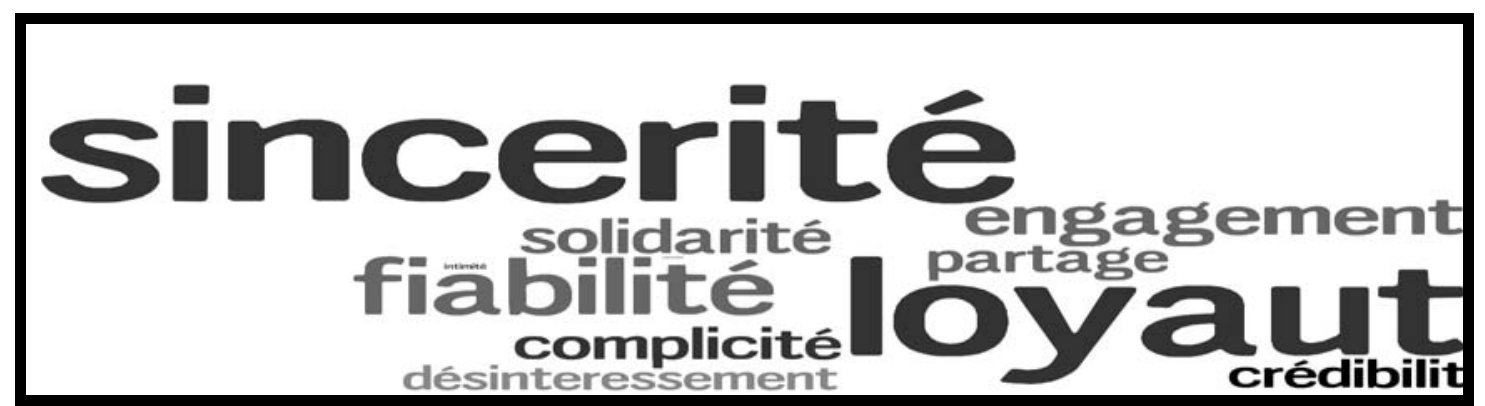

Toutefois, nous n'analyserons pas chacun des termes du tableau présenté pour immédiatement énoncer une autre définition, celle de la philosophe Michela Marzano (2011) : " [il y a dans la confiance] l'idée qu'on peut se fier à quelqu'un ou à quelque chose. [...] on remet quelque chose de précieux à quelqu'un, en se fiant à lui et en s'abandonnant ainsi à sa bienveillance et à sa bonne foi. L'étymologie du mot montre [...] les liens étroits qui existent entre la confiance, la foi, la fidélité, la confidence, le crédit et la croyance ». Définition dans laquelle l'on peut constater que réapparaissent la plupart des termes utilisés dans le tableau (ou leurs synonymes) ...comme ils

${ }^{18}$ In Encyclopédie Wikipédia (consultée le 02.07.2016).

19 Cf. les liens https://www.google.fr/search? $\mathrm{q}=$ confiance\&client=firefox$\underline{b} \&$ tbm $=$ isch \& tbo $=u \&$ source $=$ univ\&sa $=X \& v e d=0$ ahUKEwjwoLnSnNTNAhWF SwKHQoGBp0Q7AkIM A\&biw=1536\&bih=739 et $\quad$ https://www.google.fr/search?q=faire+confiance \&client=firefox$\mathrm{b} \& \mathrm{sa}=$ X\&biw $=1536 \& \mathrm{bih}=739 \& \mathrm{tbm}=$ isch \&tbo=u\&source=univ\&ved=0ahUKEwjY6Nq7ndTNAhVJWyw KHQ1zAPkQ7AkIPA (consultés le 02.07.2016). 
réapparaissent, du reste, de façon récurrente à l'écoute et la lecture de ce qui, ordinairement, se dit et s'écrit sur le thème.

Présentons également - et pour mémoire, car elles n'apportent finalement que peu d'informations supplémentaires - un ensemble de définitions issues du Dictionnaire de l'Académie Française, et dont le complexe de signification composé renvoie principalement - et pareillement -, aux idées d'espérance, de sécurité et d'assurance.

\section{Définitions de « Confiance »}

(issues du Dictionnaire de l'Académie Française, 1986)

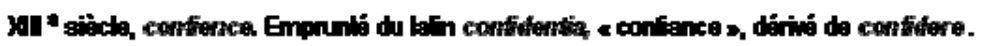

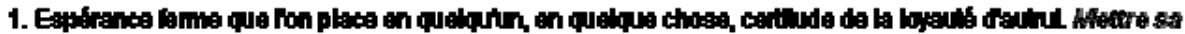

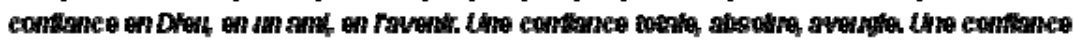

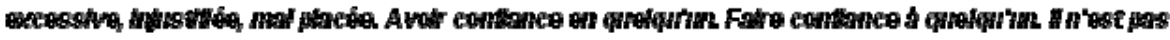

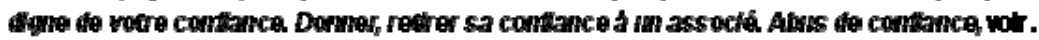

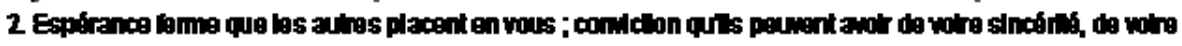

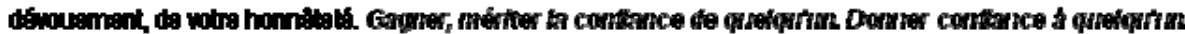

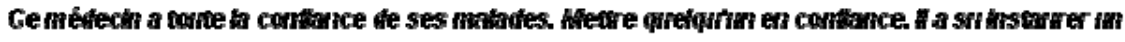

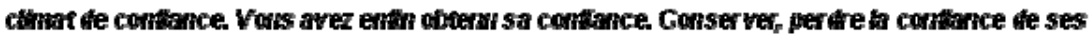

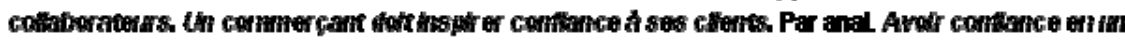
renof to

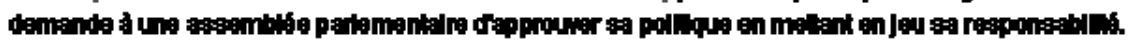

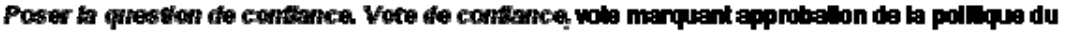
gouramemant

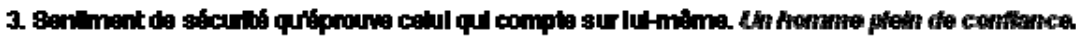

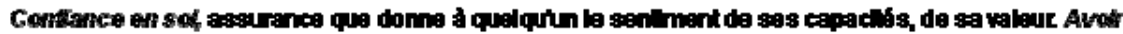
contance en sod II manpre te contance en isd

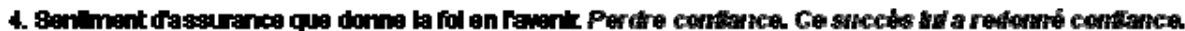

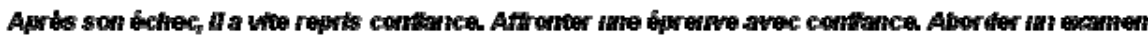

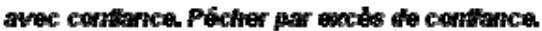

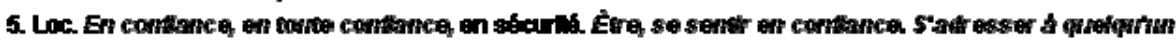

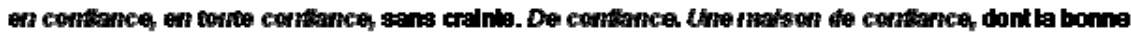

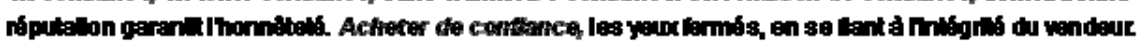

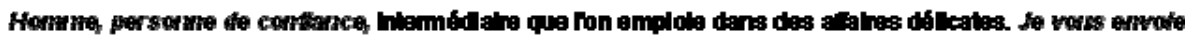

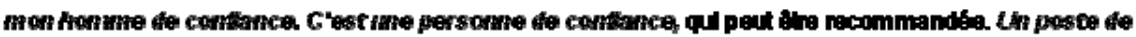

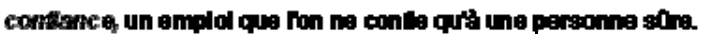

Pour compléter ce premier point relatif aux significations et définitions les plus communes du terme, et pour préciser la problématique qu'il induit dans les champs d'expérience les plus divers et variés, formulons à présent quatre exemples pratiques.

Premier champ d'étude : la confiance, disons, « en général » et comme thème central de la société contemporaine. C'est le groupe La Poste qui a lancé, dès 2008, son «Observatoire de la Confiance» en collaboration avec l'Institut TNS SOFRES ; observatoire qui étudie « ce thème central de la société contemporaine [...] en explorant quatre dimensions: confiance en soi, en les autres, dans les institutions et dans l'avenir». 


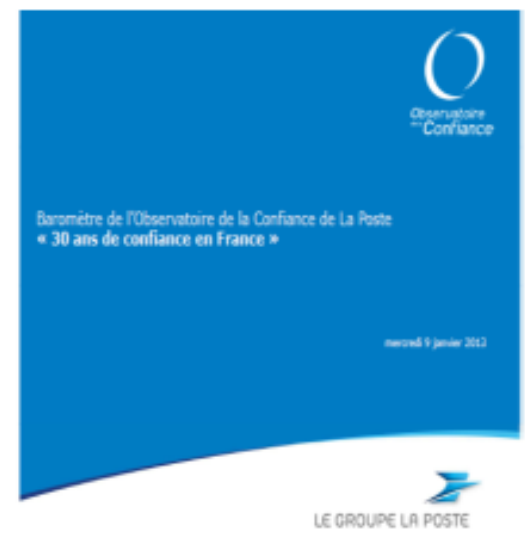

Tiers de confiance dans le monde des échanges, La Poste a créé en 2008 l'Observatoire de la Conflance.

L'objet de cette structure de recherche, de reflexion et d'action est de produire des analyses et. d'animer le débat sur ce thème central de la société contemporaine.

L'Observatoire a pour vocation de mieux comprendre les leviers constitutifs de la confiance en explorant quatre dimensions : conflance en sol, en les autres, dans les institutions et dans I'avenir. Et les trois composantes de la confiance (bienveillance, intégrité, compétence). Chaque année, le Baromètre de la confiance mesure le niveau de confiance des Français sur ces grands indicateurs.

Ce barométre est complette par une approche thématique permettant d'explorer en profondeur un des grands chantiers de la confiance de transformation de notre société. Ceci afin de connecter le barométre à l'actualité et lui conférer une utilité sociale immédiate.

Le chantier choisi en 2013 est celui de la confiance dans le Progrès.

Voir le lien:

http://legroupe.laposte.fr/Profil/L-

Observatoire-de-la-Confiance/Le-

Barometre-de-la-confiance

Grâce à une sorte de " baromètre », il s'agit de mesurer le " niveau de confiance » des français sur ces quatre indicateurs, en le complétant par une approche thématique différente chaque année - par exemple, en 2013, a été choisi le thème « Confiance dans le progrès » et en 2014 a été réalisée une mise en perspective de la confiance dans cinq pays européens (France, Espagne, Italie, Allemagne et Royaume-Uni) cherchant à situer les Français parmi leurs voisins européens en termes de confiance.

L'étude est menée selon ce qui est considéré - si on se réfère au champ sémantique (soit l'ensemble des sens disponibles du mot selon le contexte) du terme comme les «trois composantes» clefs de la confiance. A savoir la bienveillance, l'intégrité et la compétence. Ce faisant, est alors utilisée une méthode manifestement rigoureuse, fondée sur des sondages à base d'échantillons représentatifs de la société française.

Cantonnons-nous à noter que c'est la Direction de la Communication du groupe qui est en charge de l'exercice. Ce qui, selon nous, dénote un indice fort des liens étroits établis entre communication «stratégique» (voir infra) et confiance : en l'occurrence, il s'agit vraisemblablement ici d'étudier la confiance sous tous ses aspects avec l'objectif, pour La Poste, à la fois de se montrer aux yeux de tous comme un groupe responsable, analysant rigoureusement un aspect crucial de la "relation client» et en qui, justement, on peut avoir confiance relativement à ses missions de service public ${ }^{20}$, mais aussi (les deux points étant probablement intimement liés) de se servir - alors en tant qu'entreprise soumise à une obligation de résultats en termes économiques - des conclusions des travaux menés pour susciter la confiance dans le public. Autrement dit, tout se passe ici comme si l'on étudiait la confiance à la fois pour se prévaloir de l'étude et parvenir à se

${ }^{20} \mathrm{Cf}$. le lien http://legroupe.laposte.fr/Profil/Les-missions-de-service-public/Le-contrat-de-service-public (consulté le 03.07.2016). 
montrer digne de confiance auprès des consommateurs. D'une certaine manière, une stratégie ce communication comme une autre, donc...

Deuxième champ d'étude : la confiance dans les médias. Et c'est encore un baromètre annuel qui est proposé, depuis la fin des années 80 (notamment), par le quotidien La Croix (toujours avec le concours de l'Institut TNS SOFRES) ${ }^{21}$.

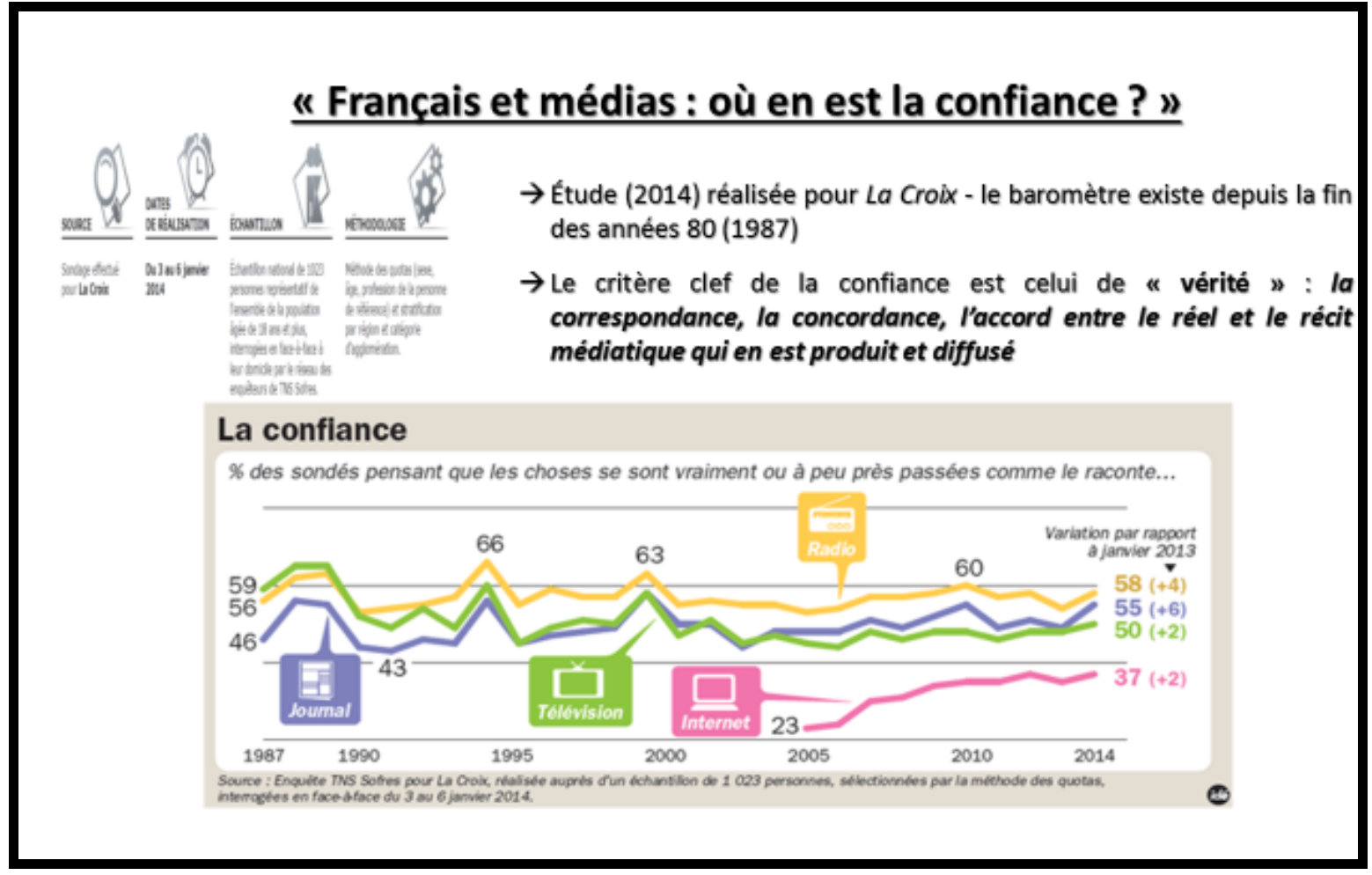

A propos de «ce[s] sondage[s] [...] censé[s] mesurer " la confiance des Français dans leurs médias" ", limitons-nous à succinctement rapporter ici ce qu'affirme le sociologue Patrick Champagne (2001, passim) : «ce " baromètre " [...] applique à la presse elle-même la logique des sondeurs qui est plus politique (on fait voter les citoyens) et économique (on cherche à mesurer la demande des consommateurs) qu'intellectuelle (est-ce que l'information est exacte et bien hiérarchisée ?) [...] Il faudrait [...] que la profession cesse de croire en la scientificité des chiffres sortis des questionnaires et s'intéresse au travail de recueil des données et à toute la logique qui est au principe de leur présentation. Bref, il faudrait que les journalistes comprennent que les instituts de sondages ne recueillent pas la réalité mais la construisent au terme d'opérations successives : sélection de la population qu'ils parviennent à interroger, formulation des questions qui sont posées, addition de réponses formellement plus que réellement identiques et grille de lecture que les sondeurs proposent des distributions statistiques à leurs commanditaires. Autrement dit, au lieu de s'interroger sur "la confiance des

\footnotetext{
${ }^{21}$ Nous présentons ici le graphique pour l'année 2014 (qui nous est apparu particulièrement clair et précis), mais pour l'année 2016 voir le lien http://www.tns-sofres.com/publications/barometre-2016-de-confiancedes-francais-dans-les-media (consulté le 03.07.2016).
} 
Français dans les médias ", il serait sans doute plus important que les journalistes s'interrogent sur les raisons de leur confiance excessive à l'égard des instituts de sondage ».

Et encore, pour continuer : "Les enquêtés répondent, dans les sondages d'opinion, à la question qu'ils comprennent et non à celle qu'on leur pose. Si l'on considère, par exemple, la question suivante qui est posée régulièrement dans le baromètre : "A quelle chaîne de télévision (ou station de radio) faites-vous le plus confiance comme source d'information?", il est probable que cette question a été, de fait, retraduite par une large fraction de la population des enquêtés, par la suivante: "Quelle chaîne (ou quelle station de radio) regardez-vous (ou écoutez-vous) habituellement? " comme semble l'indiquer la distribution des réponses qui est très proche des taux d'audience des chaînes (et des stations de radio) relevés par ailleurs dans les enquêtes de Médiamétrie. On dira qu'il y a là une nuance sans importance. En fait, le sondage transforme un simple " indice de fréquentation " en " indice de confiance", des réponses sur des pratiques en jugement sur l'excellence journalistique de tel média ou de telle chaîne. Les sondeurs font comme si les enquêtés répondaient: "je fais confiance à telle chaîne, et uniquement à telle chaîne, comme source d'informations" (sous-entendu : au terme d'un examen de l'ensemble de l'offre proposée par l'ensemble des chaînes) alors qu'il ne s'agit pour une large part que d'une simple déclaration très simplifiée sur une habitude d'écoute qui n'implique pas nécessairement de jugement particulier sur la qualité de l'information et qui ne tient pas compte, en outre les pratiques irrégulières ou plurielles ou de la pratique du zapping qui affecte l'information comme n'importe quel programme ».

Nous pensons que ce qu'affirme ici Champagne illustre bien la difficulté qu'il y a à cerner à la fois ce que l'on nomme ordinairement «confiance » et ce que l'on mesure comme tel...

Autre champ d'étude, la confiance dans la politique, proposé (particulièrement) par le CEVIPOF, le Centre d'études de la vie politique française, aujourd'hui Centre de recherches politiques de Sciences Po. Ici encore un baromètre, jusqu'ici composé de sept vagues $^{22}$ qui ont exploré, sur des échantillons représentatifs de la population française, des thèmes spécifiques en rapport avec la confiance désignée comme «la valeur cardinale de la démocratie $»$.

\footnotetext{
${ }^{22}$ Nous présentons les six premières vagues. Pour la septième (janvier 2016), axée sur la résilience, voir le lien http://www.cevipof.com/fr/le-barometre-de-la-confiance-politique-du-cevipof/resultats-1/vague7/ (consulté le 03.07.2016). Et pour une mise à jour des données, voir notamment le lien : http://www.sciencespo.fr/cevipof/fr/content/les-dynamiques-politiques-2014-2017 $\quad$ (consulté le
} 23.11.2018). 
Le CEVIPOF/CNRS a lancé depuis 2009, en partenariat avec l'institut Pierre Mendès France puis avec le Conseil économique, sacial et environnemental, son Baromètre de lo confiance politique. Cet instrument de référence compte d̀ ce jour six vagues.

\section{Le Baromètre de la confiance politique (et plus largement : en quoi les Français ont-ils conflance aujourd'hui ?)}

$1^{\text {ère }}$ vague (2009) : montre la distinction qu'opéraient les personnes interrogées : s'agissant de leurs situations personnelles $\rightarrow$ relative confiance dans l'avenir / en revanche, opinion sévère vis-à-vis du fonctionnement de notre démocratie et pessimiste face à l'avenir de notre pays.

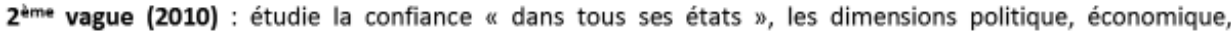
institutionnelle, sociétale et individuelle de la confiance.

$3^{\text {ime }}$ vague, (2011) : le niveau de confiance politique des Français à la veille des élections de 2012. Les résultats de l'enquête sont particulièrement instructifs de la confiance personnelle et dans la société, dans les institutions et le personnel politique.

$4^{\text {ime }}$ vague, (2012) : le niveau de confiance et les attitudes politiques des Français sept mois après le changement de majorité. Elle montre un pessimisme qui entache les conceptions de l'avenir.

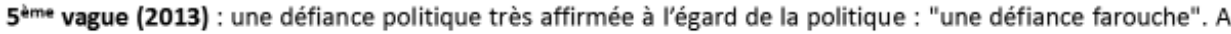
la veille des élections municipales de 2014, le Maire et le Conseil municipal sont les seuls parmi le personnel politique à bénéficier de la confiance des personnes interrogées.

$6^{\text {ìme }}$ vague, (2014) : des relations complexes entre confiance personnelle et défiance politique. Une vague supplémentaire, la vague 6 bis, capte les effets des événements de janvier 2015 .

En fait, les études menées s'intéressent d'une façon large, aux «niveaux de confiance accordée aux différents acteurs politiques, sociaux et économiques par les français ». Par exemple, la vague 5 de décembre 2013 est composée (notamment) de sections intitulées « La confiance personnelle et dans la société », « La confiance dans les institutions » ou « La confiance dans les acteurs politiques ».

L'on y découvre :

- que $10 \%$ seulement des personnes interrogées ont un état d'esprit caractérisé par ce qu'elles pensent être de la confiance :

\section{État d'esprit actuel (1/2)}

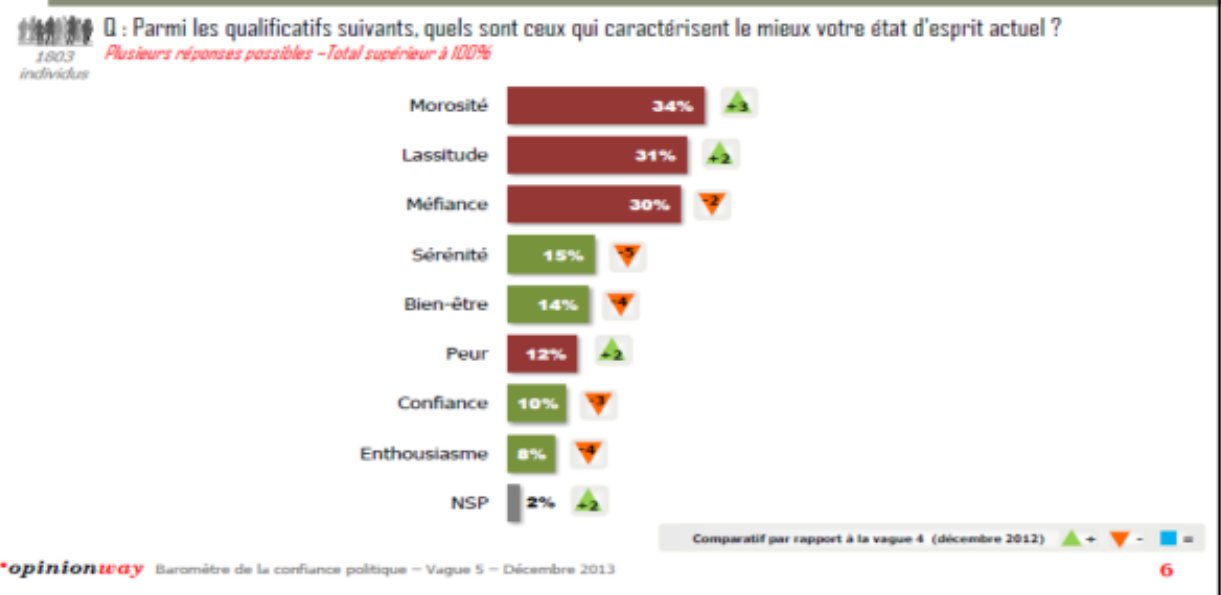


- que $75 \%$ de ces personnes sont, disons, plutôt méfiantes envers les autres :

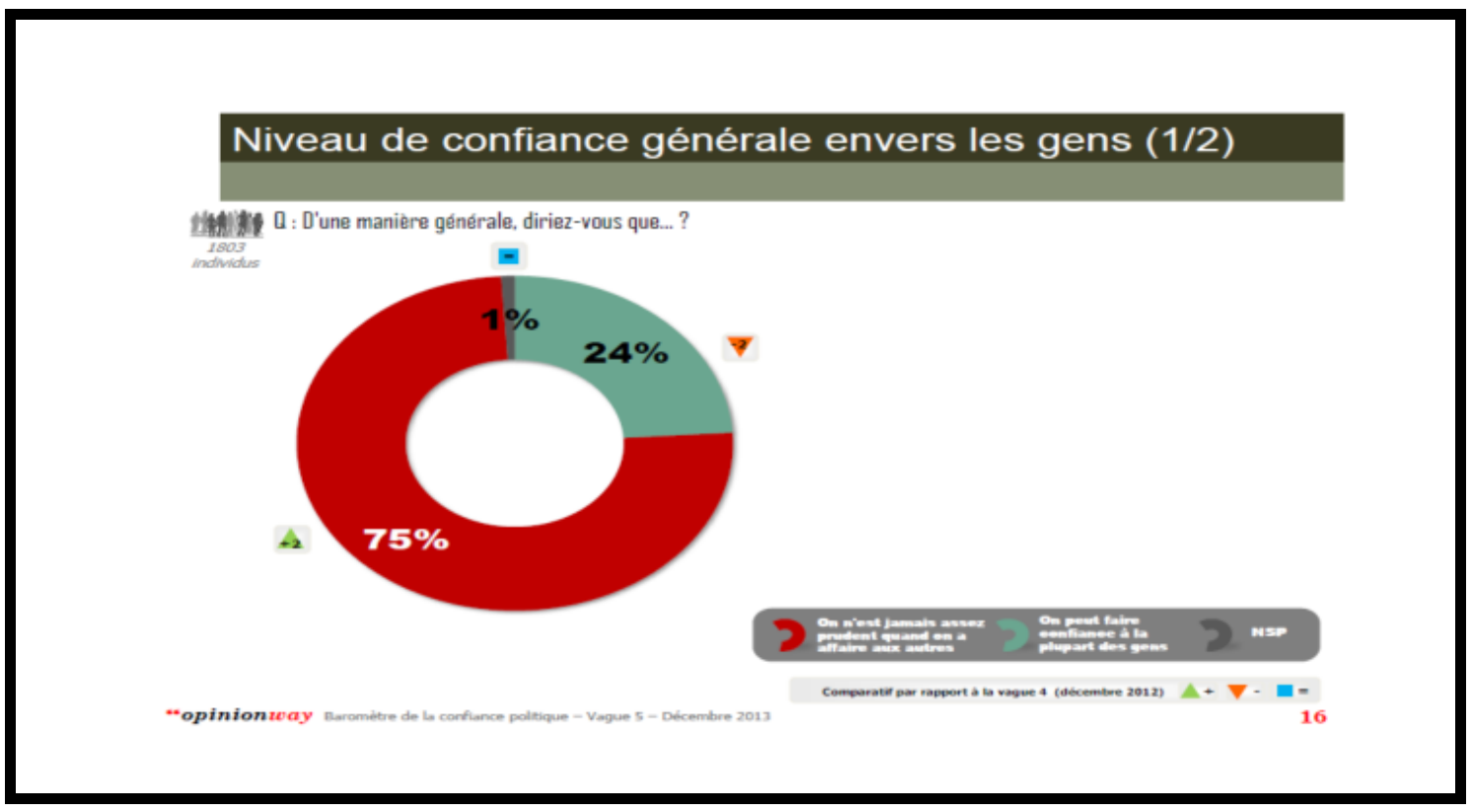

- ou encore que les personnes interrogées sont, dans l'ensemble, plutôt méfiantes envers les grandes entreprises publiques et privées, les banques, les syndicats, les médias et les partis politiques :

\section{Niveau de confiance dans certaines organisations (1/2)}

vish: Q: Avez-vous très confiance, plutôt confiance, plutôt pas confiance ou pas confiance du tout dans chacune des 1803
individus organisations suivantes...?

$$
\begin{gathered}
\text { Les hôpitaux } \\
\text { L'armée }
\end{gathered}
$$

La police

L'école

Les associations

L'Eglise catholique

La justice

Les grandes entreprises publiques

Les grandes entreprises privées

Les banques

Les syndicats

Les medias

Les partis politiques
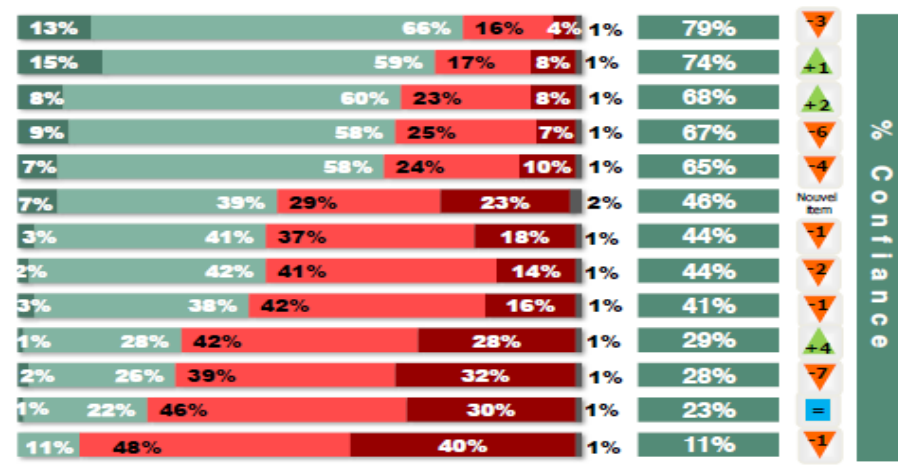

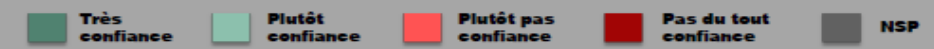

*opinionway Baromètre de la confiance politique - Vaque 5 - Décembre 2013 
Enfin, et sans aucun doute plus trivial mais économiquement très parlant: le «Contrat de Confiance» proposé par l'entreprise Darty, qui réfère au thème de la confiance envisagé par le marketing : comment une entreprise peut-elle la susciter chez ses clients? En bref, comment réaliser ce que l'on nomme une «relation client» performante, comment fidéliser une clientèle?

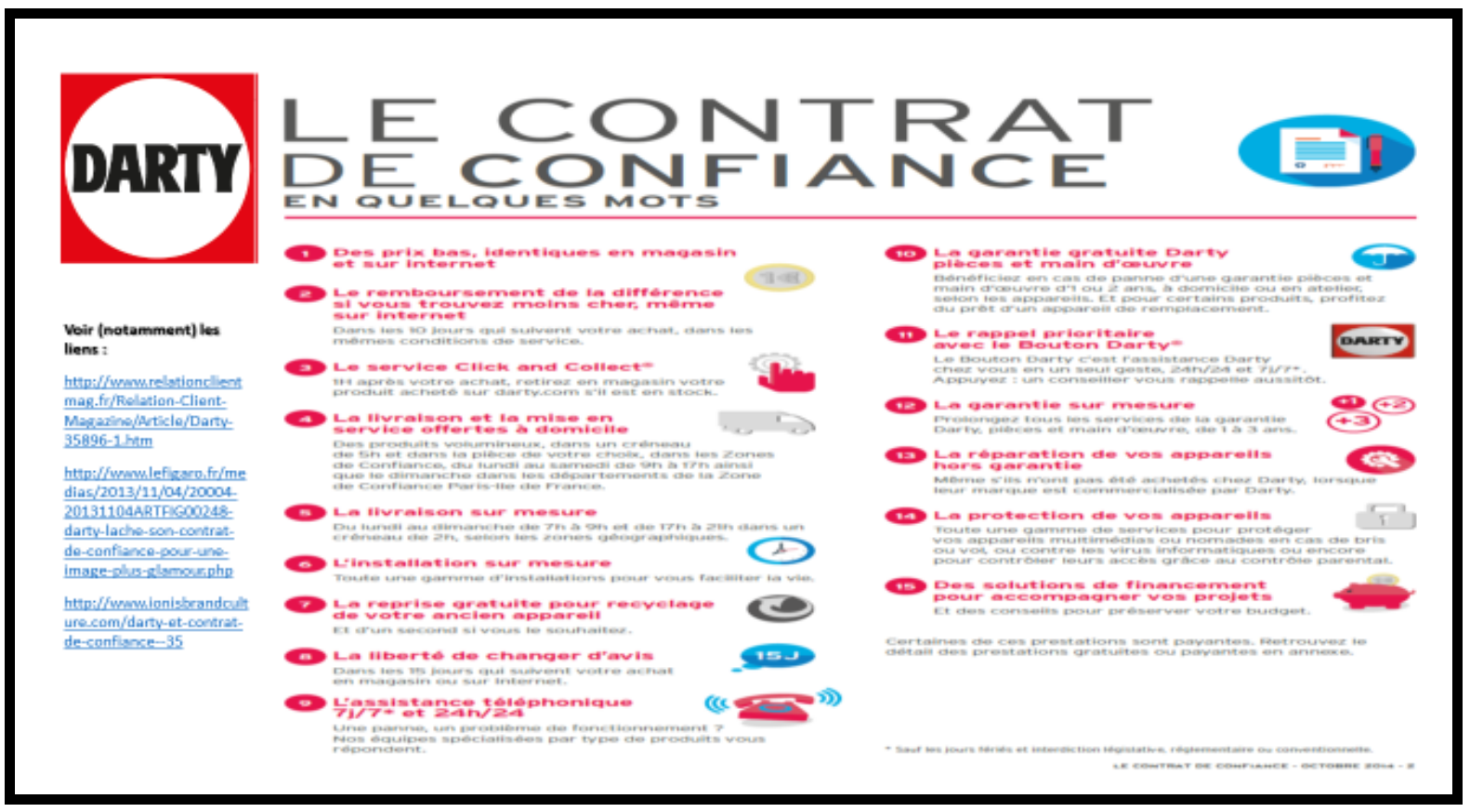

Bornons-nous à indiquer que quand bien même ce «contrat de confiance » est quelque peu passé de mode aujourd'hui - il date de 1973 -, et n'est plus mis (en quelque sorte) « en tête de gondole » par la marque - la stratégie s'axe plutôt aujourd'hui, avec la concurrence de la vente sur Internet, sur les prix bas -, il est toujours à l'ordre du jour. Et que, selon l'ensemble des experts de la matière, l'engagement (envers le client) et la garantie (du prix, du choix et des services) fournis dans le cadre tracé par ce contrat ont fondé la crédibilité de la marque, et permis à Darty de devenir leader du marché dans son domaine (l'électroménager et le multimédia).

Nous fondant sur ces quelques cas d'espèce, posons à présent la question de savoir comment, de façon plus spécifique, cerner les - ou tresser des - liens entre confiance et communication.

\subsection{Confiance, interaction, communication}

Nous nous limiterons à aborder ici deux aspects, clefs selon nous, d'un tel thème :

- d'abord, la question d'une éthique de la communication, de la relation qui, d'un certain point de vue, entretient des rapports étroits avec la question de la confiance ; 
- ensuite, cette problématique de la confiance considérée sous ses aspects à la fois rationnels et non parfaitement rationnels ...et il conviendra de s'interroger sur ce que l'on entend exactement lorsque l'on utilise le terme « rationnel».

\subsubsection{Confiance et éthique de la communication}

Très généralement, le terme «information »- certes polysémique, puisque il renvoie au moins à trois types distincts d'objets que la langue anglaise désigne par trois mots différents: la donnée objective (data), la nouvelle journalistique (news) et la connaissance ou le savoir (knowledge) -, le terme « information », donc, réfère en langue française, en tout cas communément, au «Vrai sur le Réel»: usuellement, une information constitue une description objective qui, explique le sociologue Philippe Breton (Breton, Proulx, 2002, p. 40), a pour idéal d'être un «modèle réduit », le plus fidèle possible, du réel.

Or, concernant la communication - lorsqu'on comprend le terme de la façon la plus moderne, la plus ordinaire, la plus banale aujourd'hui soit, justement, l'idée d'une « transmission d'information(s)»-, il apparait assez clairement que, pour la doxa au moins, communiquer honnêtement - éthiquement et/ou moralement, donc, et nous ne distinguerons pas ici entre les deux formes - consiste également et essentiellement à (chercher à) exprimer ce «V Vrai sur le Réel ». Soit à transmettre de « l'information » sans jamais duper ou désinformer. Du reste, comment pourrait-on tromper ce faisant, puisque l'information en tant que telle apparaît constituer l'exact inverse de la mystification, en d'autres termes l'antithèse même d'une action manipulatrice et, par suite, malhonnête ?

En fait, ce type de réponse - la communication éthique et/ou morale consiste à exprimer le Vrai sur le Réel - s'inscrit dans un cadre philosophique clairement identifiable, soit celui posé par l'impératif catégorique kantien. A savoir que le propre de la valeur morale et/ou éthique d'un comportement, c'est le désintéressement et, dans son principe, toute action morale et/ou éthique doit être (ne peut qu'être) effectuée par Devoir (lui-même désigné et requis par la Raison), et non par intérêt (ou par sentimentalité) : lorsque la raison a déterminé un principe valide, il faut constamment s'y tenir par devoir, qu'elles qu'en soient les conséquences pratiques (le devoir pour le devoir). De la sorte, lorsqu'il est question de communication - comprise au sens de transmission d'information(s), donc -, ce devoir revient à l'obligation de dire (s'il s'agit de discours) ou de montrer (s'il s'agit d'images) - en tout cas, de chercher à dire ou à montrer - le Vrai sur le Réel. Et ce quelles qu'en soient les suites. Car ne pas (chercher à) dire et/ou montrer ce Vrai revient, peu ou prou, à mystifier autrui. Action qui apparaît éthiquement et/ou moralement illégitime car rationnellement incohérente - rappelons que dans le cadre kantien, la morale se fonde sur les exigences de la Raison - dans la mesure où l'autorisation de la possibilité d'un seul mensonge - de facto toujours accompli par intérêt -, quel qu'il soit et quelle qu'en soit la cause et le but, est de nature à annihiler définitivement la crédibilité de toute parole en général.

Autrement dit, si je tolère la possibilité d'un seul mensonge, comment ma parole, alors possiblement mensongère, pourrait-elle être digne de confiance ? En fait, dans cette approche, elle ne le pourrait plus, et à jamais...

En bref, sauvegarder des relations humaines harmonieuses - exigence rationnelle et logique d'une vie en société - implique, avant tout, de garantir la confiance que 
s'accordent les hommes entre eux, ce qui suppose, toujours, qu'ils recherchent et énoncent la Vérité, en tout cas et au moins, « leur » vérité.

\title{
QU'EST-CE QU'UNE ÉTHIQUE DE LA COMMUNICATION ?
}

\section{Communiquer honnêtement, moralement/éthiquement, " dans le}

\author{
sens du Bien $"=>$
}

\section{(chercher à ) énoncer/montrer constamment " le Vrai sur le Réel "}

$\rightarrow$ transmettre de "l'information " (au sens de " données objectives ") sans jamais duper, "désinformer» : " devoir de vérité » du communicant débouchant sur une "éthique de vérité» (souvent dite "de conviction ")

$\rightarrow$ réponse à envisager comme issue du cadre philosophique posé par l'impératif catégorique kantien : l'éthique est fondée sur la raison, et une action " éthique " est effectuée par devoir; en l'occurrence, le devoir est toujours d'énoncer le vrai sur le réel...

\section{...sinon, la confiance relationnelle étant altérée, le lien social se trouve en grave danger de décomposition}

On découvre la position centrale qu'occupe ici la notion de confiance en matière de communication conçue comme transmission d'information(s): elle est tout simplement, en tout cas dans cette conception kantienne - qui est la plus unanimement, la plus ordinairement partagée -, au fondement même d'une éthique de la communication. Dans ce cadre, communiquer éthiquement, c'est perpétuellement chercher à dire le vrai sur le réel sans la moindre possibilité de mensonge, sans quoi le lien social, fondé sur la confiance en la parole d'autrui, viendrait à être irrémédiablement rompu...

Nous ne nous étendrons pas plus avant sur ce thème de l'éthique de la communication $^{23}$ et de ses rapports avec la confiance sinon indiquer, pour mémoire, que partageant l'optique constructiviste, il est tout à fait possible de ne pas cautionner une telle conception selon laquelle, pour résumer, la communication est éthique en soi si elle dit le Vrai - en l'occurrence, dire le Vrai, c'est aller dans le sens du Bien, puisque c'est préserver la confiance que se font les hommes entre eux, et donc pérenniser le lien social-, et immorale en soi si elle ne se cantonne pas à apporter de «l'information », à décrire le réel tel qu'il est.

En effet, selon un point de vue divergent, le «comportement adéquat » - pour utiliser une expression attribuée au philosophe Baruch Spinoza lorsqu'il définit l'éthique - qui se trouve au fondement de (ou même qui constitue) la morale et/ou l'éthique ne revient pas tant, en communication, à toujours chercher à dire la vérité sur le réel que de se poser en permanence la question de savoir quels vont être pour autrui les effets des messages qu'en tant que communicants (professionnels ou non) nous

${ }^{23}$ Pour des développements sur ce thème spécifique, voir Benoit, Ethique et Communication, 2012, passim. 
concevons et diffusons. Autrement dit, il ne s'agit plus de respecter un impératif catégorique, soit un grand principe théorique d'une irréprochable cohérence - en l'occurrence il faut, sans dérogation possible, toujours dire (ce que l'on considère être) le vrai, « informer»-, mais de tenter, avec une persévérance sans faille, d'évaluer les comportements en fonction de leurs conséquences pratiques, par définition toujours problématiques ; en d'autres termes, à mettre en œuvre l'« éthique de responsabilité » chère au sociologue Max Weber (1959, passim) pour lequel atteindre des fins « bonnes » nous oblige la plupart du temps à compter avec «des moyens malhonnêtes ou pour le moins dangereux». Dans ce dernier cadre de pensée, le non-mensonge certes possiblement producteur, à terme, de confiance, n'est plus le garant d'une éthique relationnelle, qui demande alors essentiellement aux communicants une réflexion, une interrogation constante non pas tant sur les moyens employés - mensonge ou vérité, notamment - que sur les fins poursuivies. La communication n'est dès lors plus éthique en elle-même (si elle cherche ou non à dire le Vrai), mais voit l'éthique lui être comme extérieure, "importée » dans leurs actions par des communicants attentifs aux fins éthiques ou non - qu'ils poursuivent.

\section{QU'EST-CE QU'UNE ÉTHIQUE EN COMMUNICATION ?}

\section{Communiquer honnêtement, moralement, « dans le sens du Bien »}

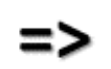

- mettre en œuvre un " comportement adéquat» (Spinoza) $\rightarrow$ pour l'essentiel à se poser la question de savoir quels vont être les effets pour les autres des messages que je conçois et que je diffuse : "cette seule interrogation [...] suffit à initier une éthique ", (Tessier, 2004) $\rightarrow$ "L'éthique ne sera jamais un mode d'emploi. L'éthique ne fait qu'interroger, interroger sans cesse les fondements ", (Sicard, 2001)

- mettre en œuvre une " éthique de responsabilité » ou Verantwortungsethik : " Pour atteindre des fins 'bonnes', nous sommes la plupart du temps obligés de compter avec, d'une part, des moyens malhonnêtes ou pour le moins dangereux, et d'autre part, la possibilité ou encore l'éventualité de conséquences fâcheuses ", (Weber, 1959)

Concluons en précisant qu'une telle position apparaît aujourd'hui minoritaire. Et que de nombreux auteurs - par exemple, en sciences de l'information et de la communication, le concepteur de la méthode «PAT-Miroir», Gilles le Cardial (1997, passim) - fondent l'éthique relationnelle sur la loyauté de la parole, concrètement sur le non-mensonge inducteur de confiance, (cette dernière assurant la stabilité de la vie en société).

\subsubsection{Une « logique » de la confiance}

Regardons à présent la confiance sous un angle, disons, " cognitif » ...mais pas seulement cognitif. En effet, à l'instar de Louis Quéré (ibid.) - qui juge qu'une conception purement cognitive de la confiance n'est pas satisfaisante -, nous 
considérerons que la confiance repose certes sur des opérations cognitives mais que, tout en se différenciant de la foi stricto sensu, elle comporte un élément de choix, de " pari », de « saut dans l'engagement » non entièrement étayé par des raisons ou des croyances.

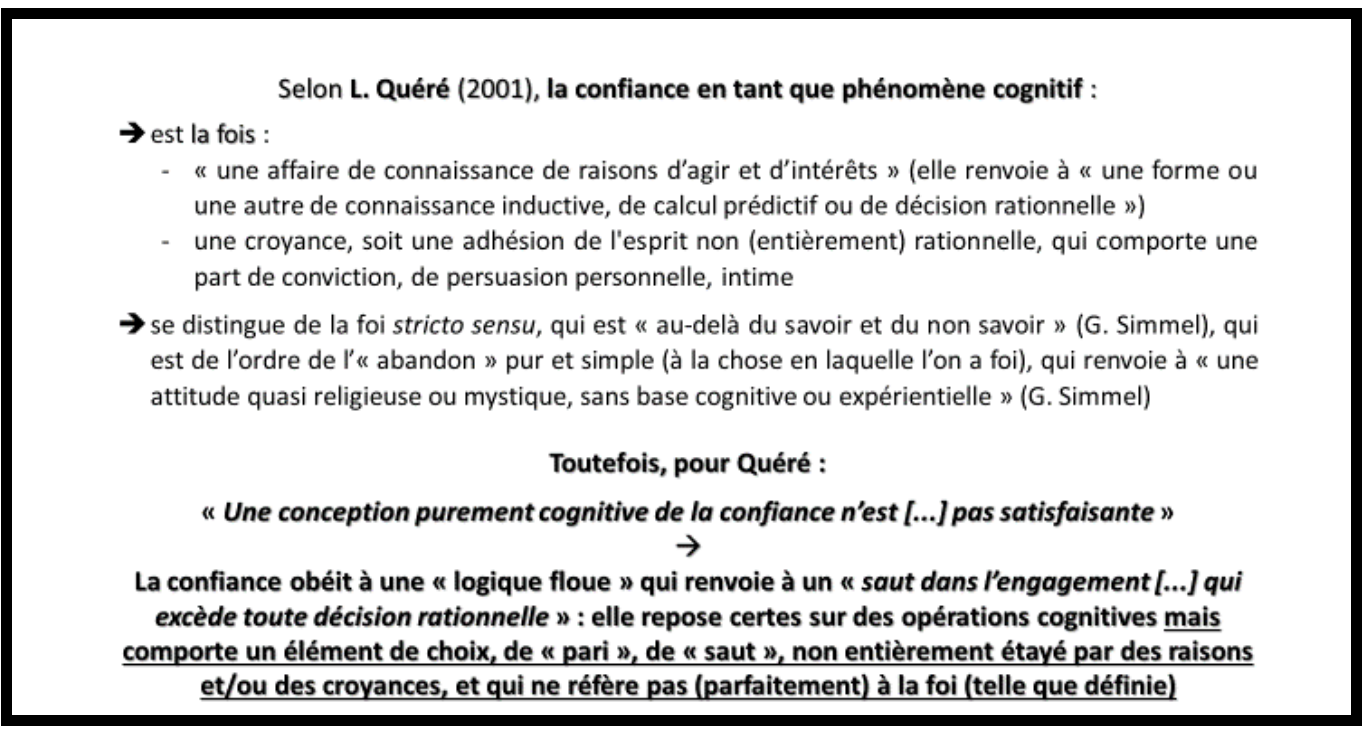

En bref, reprenant (d'une certaine façon) la formule du sociologue Georg Simmel (explicitée par Quéré, ibid.), " celui qui sait tout n'a pas besoin de faire confiance; celui qui ne sait rien ne peut raisonnablement même pas faire confiance »-ce qui, en d'autres termes, signifie, explique Quéré, que " là où il y a information complète, transparence de la situation du point de vue cognitif, il n'y a pas de place pour la confiance ; celle-ci $n$ 'intervient que s'il y a manque d'information ou de connaissance et que si tout n'est pas tenu pour allant de soi $\gg-$, en reprenant, donc, ladite formule, nous situerons la confiance « quelque part » sur un hypothétique continuum dont les deux limites seraient, d'un côté, la connaissance fondée sur le raisonnement, la statistique, les probabilités, l'expérience et, de l'autre, la foi disons « illuminée » et/ou aveugle. 


\section{CONFIANCE}

\section{Savoir \\ «Zone » de la confiance \\ Foi aveugle}

\section{" celui qui sait tout n'a pas besoin de faire confiance; celui qui ne sait rien ne peut raisonnablement même pas faire confiance "}

$\rightarrow$ une combinaison variable de savoir et de non-savoir...

Georg Simmel (1999)

Sur une telle base, référons-nous au célèbre jeu connu sous la dénomination de « dilemme des prisonniers », tel qu'explicité par Paul Watzlawick dans son ouvrage La réalité de la réalité (1978, passim).

En fait, Watzlawick utilise un tel cas d'espèce pour clarifier le concept d'« interdépendance », défini comme le fait qu'une chose dépend d'une autre qui ellemême dépend de la première. Dit autrement, l'interdépendance renvoie à ce que l'on nomme causalité circulaire : soit lorsque une cause produit un effet, qui lui-même se change en cause pour rétroagir (feed back) sur la cause initiale. Soulignons que cette causalité circulaire apparaît comme étant l'essence même de ce que l'on nomme « interaction » en matière de relations interpersonnelles.

\section{INTERDÉPENDANCE ET CONFIANCE}

\section{Le dilemme des prisonniers}

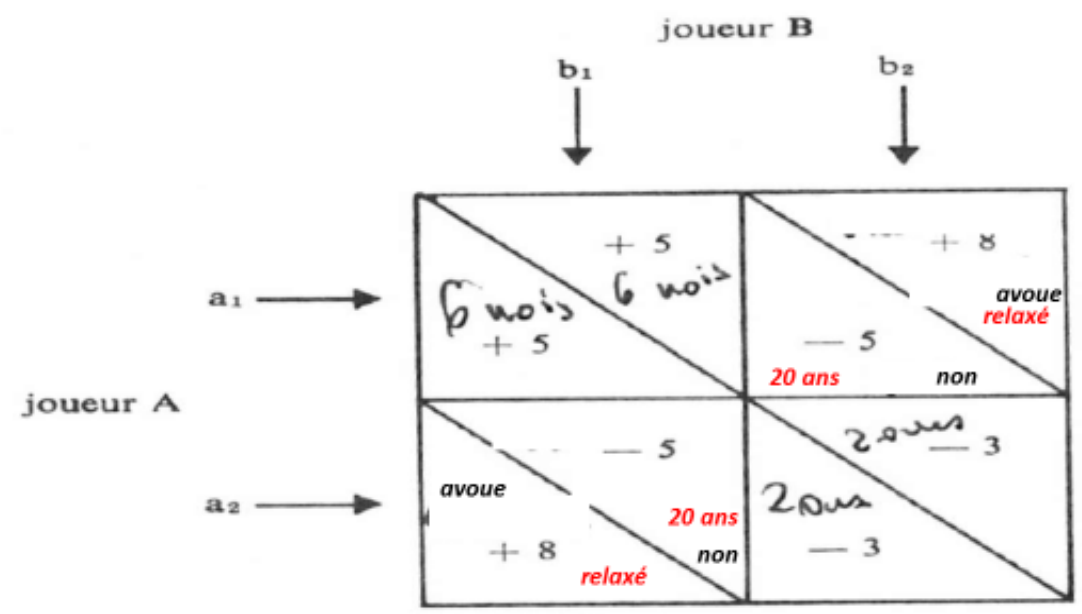

(extrait de P. Watzlawick, La réalité de la réalité, 1978, p. 102) 
Alors, lorsqu'on le découvre, l'on saisit rapidement que la question de la confiance (et de son degré) se trouve au centre même du jeu, dans la mesure où, pour les joueurs, le profit maximum est entièrement dépendant de sa présence ou de son absence : la décision la plus raisonnable et souhaitable ne peut être prise que si les joueurs se font confiance, et qu'ils résolvent ainsi le dilemme. Rappelons qu'un dilemme peut être défini comme la "nécessité dans laquelle se trouve une personne de devoir choisir entre les deux termes [...] également insatisfaisants d'une alternative » : c'est-à-dire qu'un choix doit être fait par un acteur, mais quel que soit le choix effectué, ledit acteur n'aura in fine pas pris l'option adéquate. En l'occurrence, seule la confiance possède le pouvoir de faire disparaitre le dilemme...

Usuellement, le dilemme des prisonniers est présenté sous la forme de cette matrice (ci-dessus). Reprenons-en la version originale commentée par Paul Watzlawick : « un magistrat d'instruction tient deux hommes pour suspects d'un vol à main armée. Les preuves manquent pour porter I'affaire devant les tribunaux; il fait donc convoquer les deux hommes. Il leur dit avoir besoin d'aveux pour les faire inculper ; sans quoi, il peut seulement les poursuivre pour détention illégale d'armes à feu, délit les faisant encourir une peine de six mois de prison. S'ils avouent tous les deux, il leur promet la sentence minimale pour vol à main armée, soit deux ans. Mais si un seul avoue, il sera considéré comme témoin officiel et relaxé, tandis que l'autre prendra vingt ans, le maximum. Puis, sans leur donner l'occasion d'élaborer une décision commune, il les fait enfermer dans des cellules séparées, d'où ils ne peuvent communiquer». Que faire.. ?

La meilleure solution est, d'évidence, de ne rien avouer pour les deux prisonniers (a1/b1). Toutefois, sachant que si l'un avoue et l'autre pas, celui qui n'a pas avoué sera très lourdement condamné $(\mathrm{a} 1 / \mathrm{b} 2-\mathrm{a} 2 / \mathrm{b} 1)$, la décision la plus « sûre » pour les deux, le choix le plus empreint de rationalité - et la «rationalité» est ici conçue comme renvoyant à des comportements dits «logiques », la logique étant définie comme la méthode de pensée qui permet de distinguer les raisonnements pertinents de ceux qui ne le sont pas et, en tout cas, de sélectionner les raisonnements les mieux pertinents, nous allons y revenir en détails... -, le choix le plus empreint de rationalité, donc, est sans aucun doute $\mathrm{a} 2 / \mathrm{b} 2^{24}$, car il permet (paradoxalement) de maximiser la satisfaction des deux prisonniers en minimisant, disons, leurs «pertes» potentielles, résultat tout de même très décevant, en tout cas comparé à la meilleure solution...

Autrement dit, le dilemme peut être résumé en ces termes :

1) pour moi et mon compagnon, il vaut mieux ne rien avouer ;

2) mais si je n'avoue pas et que mon compagnon avoue, j'en prends pour 20 ans (et lui rien);

3) et si j'avoue et que mon compagnon n'avoue pas, c'est lui qui en prend pour 20 ans...

4) si nous avouons tous les deux - solution la plus circonspecte - c'est 2 ans de prison ...ce qui n'est tout de même pas rien.

Relativement à notre objet de recherche, ce qu'il convient de bien saisir c'est que, dans le cadre ainsi tracé, le degré de confiance entre les deux compagnons constitue le sésame qui permet (éventuellement) de sortir du cercle vicieux : soit les deux compagnons se font mutuellement confiance, se fient l'un à l'autre, et c'est la meilleure

\footnotetext{
${ }^{24}$ Voir (par exemple) le lien http://fr.wikipedia.org/wiki/Dilemme du prisonnier (consulté le 15.07.16).
} 
solution qui peut être retenue, soit ils ne se font pas confiance (ou insuffisamment), et c'est une solution certainement très frustrante qui est mise en œuvre ; la solution « entre deux » - revenant pour l'un à rompre la confiance que l'autre, in fine complètement lésé, lui accorde possiblement - étant, également et indubitablement, insatisfaisante.

Disons que le dilemme des prisonniers a fait l'objet d'un nombre vertigineux d'investigations scientifiques, et que de nombreuses variantes en ont été proposées. Il constitue l'un des exemples les plus connus de ce que l'on nomme, à la suite des travaux à la fin des années 20 du mathématicien et physicien américano-hongrois John Von Neumann, "Théorie des jeux », soit une branche des mathématiques (bien souvent vulgarisée de façon discutable et critiquée).

A ce sujet, cantonnons-nous à rappeler qu' "une hypothèse de base de la théorie des jeux [...] est de considérer que les agents sont 'rationnels', c'est-à-dire qu'ils tentent d'arriver à la situation la meilleure pour eux », en " calculant» le choix optimal, qu'ils cherchent à "maximiser leur [...] objectif compte tenu de l'information dont ils disposent $»^{25}$.

Or, posons la question : qu'est-ce que, à proprement parler et plus précisément, la « rationalité », qu'est-ce qu'un « agent rationnel », et comment peut-il l'être (rationnel) ?

Si l'on laisse quelque peu de côté le cadre limité offert par le dilemme des prisonniers pour aborder la vie « réelle » dans son infinie complexité, l'on considère, à la suite des travaux du sociologue et économiste Herbert Simon (1976, passim), que la rationalité des acteurs n'est jamais "parfaite»- elle le serait si les agents pouvaient disposer de l'ensemble des informations nécessaires pour atteindre le meilleur résultat possible, et qu'il leur suffisait, pour ce faire, d'appliquer un raisonnement déductif valide $^{26}$-, mais que cette rationalité est «limitée» ou «affaiblie». A savoir (si l'on simplifie largement les interprétations possibles de la notion de rationalité dite limitée et leurs explications) que les individus, outre un accès imparfait aux informations relatives à un environnement qui se modifie sans cesse, ont des capacités cognitives bornées qui restreignent leur aptitude à concevoir la réalité dans sa complexité ontologique. En d'autres termes : "l'individu n'a pas les capacités cognitives nécessaires pour envisager toutes les conséquences possibles de sa prise de décision, associer des probabilités à ces différentes conséquences et événements futurs, tenir compte de sa propre méconnaissance de certaines informations, intégrer les comportements stratégiques des autres acteurs, calculer l'utilité espérée qu'il retirerait de ces différentes situations, et, enfin, choisir la décision qui lui apporterait le plus de satisfaction » (Béjean, Midy, Peyron, 1999, passim).

Toutefois, Simon introduit un autre concept, celui de « rationalité procédurale ». Concept qui apparaît particulièrement intéressant relativement à la question de savoir comment se génère cette confiance, ô combien cruciale en matière interactionnelle, et s'il est possible d'en établir ou favoriser (et comment) l'émergence, puisque l'on sait, pour y revenir, que pour résoudre le dilemme des prisonniers "quelque chose d'autre » que la logique et la raison doit entrer en jeu. Et ce quelque chose, à savoir la confiance mutuelle, possiblement « déraisonnable » ou non parfaitement « logique », ne peut être ni imposé ni

25 Cf. le lien http://www.cril.univ-artois.fr/ konieczny/enseignement/ TheorieDesJeux.pdf (consulté le 15.07.16).

${ }^{26} \mathrm{Cf}$. les liens http://mikael.cozic.free.fr/rationalitelimitee.pdf, http://leg.u-bourgogne.fr/documents-detravail/e9914.pdf (consultés le 15.07.16). 
provoqué délibérément comme le soutient Paul Watzlawick, qui précise que : "même si les prisonniers trouvaient le moyen de communiquer pour prendre une décision commune, leur sort dépendrait encore du degré de confiance qu'ils ont I'un en l'autre : sans confiance, le cercle vicieux ne cessera de recommencer $\gg$.

En effet, pour Simon, la rationalité des acteurs ne doit pas (ou plus) être considérée sous l'angle d'un simple calcul trivial « risque-intérêt », comme consistant, pour eux, à viser grâce à la mise en œuvre d'un raisonnement (de type déductif, formel) un meilleur résultat possible, donné, déterminé a priori. Mais, l'environnement étant indéterminé, parce que les acteurs le construisent eux-mêmes au fur et à mesure de leurs actions, la rationalité se situe dans la cohérence, la congruence du raisonnement (alors de type délibératif ou argumentatif) qui conduit à la décision.

Ainsi, la rationalité de l'individu ne se trouve plus seulement dans la conséquence attendue de la décision, soit l'utilité ou le profit optimal calculé arithmétiquement dans le cadre d'un environnement qui existe en dehors de lui-même (rationalité dite " exogène), mais "s'exerce dans la recherche d'une procédure apte à définir à la fois l'objectif recherché et les moyens d'y parvenir » (rationalité dite " endogène »), les objectifs de l'action se construisant pendant le processus de décision qu'effectue en lui-même l'individu.

En d'autres termes, la rationalité procédurale " n'a [...] pas à être jugée dans un ajustement des moyens aux fins puisque ces fins ne sont pas prédéfinies » et, explique Simon (1996, passim), "le problème se déplace graduellement du choix de la meilleure façon d'agir (rationalité substantielle) à la recherche du moyen de déterminer, de manière très approximative, où se situe une bonne façon d'agir ».

\section{Divers types de rationalité \\ (selon Herbert SIMON, 1955, 1976, 1996)}

dite « parfaite $n$ ( $\propto$ substantielle $w$ ou « substantive $n)$ : lorsque les agents peuvent disposer de l'ensemble des informations nécessaires pour atteindre le meilleur résultat possible (en appliquant un raisonnement de type déductif)

dite "limitée " : « l'individu n'a pas les capacités cognitives nécessaires pour envisager toutes les conséquences possibles de sa prise de décision, associer des probabilités à ces différentes conséquences et événements futurs, tenir compte de sa propre méconnaissance de certaines informations, intégrer les comportements stratégiques des autres acteurs, calculer l'utilité espérée qu'il retirerait de ces différentes situations, et, enfin, choisir la décision qui lui apporterait le plus de satisfaction w

dite " procédurale n: « il n'y a plus une, et unique, décision rationnelle, mais des manières rationnelles de prendre les décisions en fonction des informations sélectionnées, de l'évolution de l'environnement et en fonction de valeurs qui peuvent également évoluer $\$$; la rationalité de l'individu s'exerce dans la recherche d'une procédure apte à définir, à la fois et conjointement, l'objectif recherché, les moyens d'y parvenir et les valeurs subjectives qui le motivent ; l'individu ne sait pas précisément quel résultat il vise (il ne connait pas a priori le choix optimal « pour lui-même $»)$ : les objectifs de son action ne sont pas prédéterminés mais se construisent pendant l'action, pendant le processus de décision ; il ne s'agit plus d'un simple utilitarisme (prégnant dans les deux autres types de rationalité), à savoir chercher à obtenir par calcul le meilleur résultat " pour soi », mais l'individu peut intègrer des motivations de toutes sortes (par exemple, préférer l'équité, l'intérêt général ou l'altruisme à son propre intérêt, remplacer la défiance par la confiance, etc.)

d’après S. Béjean, F. Midy, C. Peyron (1999)

L'on peut alors considérer que les conséquences de l'application de ce modèle de la rationalité procédurale sont les suivantes (nous reprendrons intégralement ici les conclusions de Béjean, Midy et Peyron, ibid.). Le modèle : 
- permet d'envisager un environnement décisionnel évolutif qui se construit au gré des actions et de l'imagination des hommes. Il n'y a plus une, et unique, décision rationnelle, mais des manières rationnelles de prendre les décisions en fonction des informations sélectionnées, de l'évolution de l'environnement et en fonction de valeurs qui peuvent également évoluer ;

- autorise une plus grande richesse cognitive en multipliant les moyens d'accéder à la décision. Ceux-ci ne se limitent plus au calcul [...] mais s'étendent à la délibération et au respect [...] de normes sociales, de modèles de comportement; ainsi la rationalité procédurale autorise-t-elle une plus grande richesse cognitive que la simple mise en œuvre d'une règle de calcul ; en cela, les capacités cognitives des individus ne sont pas limitées mais au contraire plus étendues et plus complexes ;

- autorise une plus grande liberté quant au critère de décision à retenir : puisque dans le monde réel l'optimum ne peut plus servir de norme décisionnelle, l'acteur économique accepte des alternatives qu'il juge suffisamment bonnes en fonction d'un niveau d'aspiration qui lui est propre; les critères de décision ne peuvent alors plus faire référence à l'optimalité du résultat de la décision, mais à la satisfaction des procédures mises en œuvre ; ce faisant, l'objet de la rationalité s'est déplacé du résultat « décision » à l'action de « décider»;

- enfin, quant aux valeurs qui motivent l'action, elles sont diverses et irréductibles au pur individualisme de l'hypothèse de rationalité substantielle : en autorisant le respect de valeurs altruistes, désintéressées, ou collectives, on élargit la conception même de l'individu qui n'agit plus dans un univers désincarné, dépourvu de lien social, mais s'intègre au contraire dans une société où des normes sociales guident également les comportements, en plus de valeurs plus individualistes.

Cet ensemble de conséquences - outre qu'il renvoie directement aux thèses constructivistes, selon lesquelles le réel n'existe pas «en soi», ne nous est pas «extérieur» mais se construit dans une interdépendance constante avec nos propres actions, qu'il fait irrésistiblement penser à la conception défendue par le neuroscientifique portugais Antonio Damasio (1995) selon lequel, contrairement à ce qu'allègue une certaine culture classique cartésienne, les émotions, qui nous permettent de nous adapter à l'environnement, font partie intégrante de la raison (d'où le titre évocateur de son ouvrage, L'erreur de Descartes), et qu'il évoque la théorie des «stades moraux » de Lawrence Kohlberg (1958), selon lequel au stade 6, le plus élevé, il n'est plus question d'agir en fonction de ce que les autres veulent ou de la récompense attendue, mais selon des principes éthiques de son propre choix définis par la réflexion -, cet ensemble de conséquences, donc, semble bien permettre de considérer que la confiance, souvent superfétatoire lorsqu'il s'agit de calculer «logiquement», « rationnellement» (au sens ordinaire de ces termes) notre intérêt dit «optimal », peut être intégrée à bon escient dans le calcul ...qui change du reste de nature pour devenir une délibération complexe, empreinte des valeurs qui nous animent. En d'autres termes, notre « intérêt» se complexifiant, la confiance qui peut apparaître si " déraisonnable » dans une approche disons strictement et trivialement «mathématique» du réel et de l'interaction humaine, qui peut apparaitre comme un simple «avatar de l'intérêt» (Thuderoz, Mangematin, Harrison, 1999, passim), devient une option tout à fait crédible, intéressante, et le plus souvent si parfaitement efficace qu'il convient probablement de ne jamais en négliger l'occurrence. Même si, de fait, la question de la pure « efficacité » est 
ici dépassée puisque la confiance devient ainsi une affaire de valeurs, à traiter sur un plan bien plus axiologique que pragmatique. Dans ce sens, Paul Watzlawick (ibid.) propose l'exemple suivant : "au cours des pourparlers Nixon-Brejnev de juin 1974, le gouvernement des États-Unis montra clairement son intention de ne pas construire la base de missiles antibalistiques (MAB) prévue aux termes du traité de 1972 sur I'armement nucléaire. Face à cette décision unilatérale, les Soviétiques se sentirent, semble-t-il, suffisamment en confiance pour renoncer à la construction de leur seconde base MAB [...]. Cet épisode fut à juste titre accueilli comme un tournant historique dans les relations entre les deux pays ; il n'a été rendu possible qu'après qu'une des deux parties eut unilatéralement abandonné le langage de la pure rationalité, pour avancer d'un pas dans la confiance que I'autre partie n'en tirerait pas avantage. Les États-Unis se sont, autrement dit, exposés à tous les risques comportés par la confiance et qui la rendent si 'déraisonnable'»...mais, conclurons-nous, le résultat terminal en a été considérablement bénéfique.

Si la confiance, au delà du simple calcul « utilitaire », borné et obtus, est ou peut être fructueuse à établir quelque soit le domaine des relations humaines concerné et sur des plans très différents, comment en favoriser l'émergence en utilisant la communication? Quand bien même les deux cadres (interactionnel et organisationnel) peuvent se révéler étroitement liés - notre hypothèse est que les liens interindividuels exercent, en deçà des structures propres aux organisations, une influence sur ces mêmes structures, autrement dit que les structures organisationnelles apparaissent dépendantes des interactions, et inversement (Bernoux, 2004, passim), confer l'ensemble des études «systémiques », et particulièrement celles dédiées au principe d'homéostasie, selon lequel le fonctionnement et la nature d'une structure peuvent être profondément transformés par l'action des, ou sur les, éléments singuliers qui la composent (Benoit, 2013, pp. 65-80) -, quand bien même les deux cadres peuvent se révéler étroitement liés, donc, ce type d'interrogation élargit inévitablement la problématique, et tend à nous faire passer d'un questionnement axé sur les relations interpersonnelles, l'interaction, à un autre, plus large, référant aux systèmes, aux organisations ${ }^{27}$.

\subsection{Confiance, communication, organisations}

D'emblée indiquons que, dans les quelques réflexions que nous nous limiterons à proposer ci-dessous sur ce thème, nous comprendrons la locution « la communication » comme référant à la fois au modèle «de la transmission» et à celui « de la participation » théorisé par l'Anthropologie de la communication (Winkin, 1996, passim). Dernier modèle qui conçoit celle-ci comme « matrice sociale », communiquer ne revenant alors pas tant à transmettre stricto sensu des informations (selon le schéma rebattu Emission-Réception) qu'à participer à une culture, à une organisation sociale : les

\footnotetext{
${ }^{27}$ A noter que les diverses recherches sur le thème de la confiance portent aujourd'hui sur trois principaux types différenciés, à savoir: la confiance interpersonnelle, qui prend place entre des individus indépendamment de leurs titres ou de leurs postes ; la confiance organisationnelle, qui réfère à l'« action collective » des membres engagés dans le fonctionnement d'un organisme ; et la confiance sociale ou institutionnelle, qui est basée sur une structure sociale « formelle », soit le cadre juridique et réglementaire qui régit chaque société (selon Maurel, Chebbi, 2012, passim).
} 
messages transmis ne peuvent être interprétés et compris que mis en rapport avec leur contexte de transmission. Ce faisant, les rapports entre confiance et communication peuvent en effet être appréhendés dans toute l'étendue de leurs différentes dimensions. Soit à la fois comme renvoyant à une forme d'apprentissage effectué sur la base d'informations qu'autrui nous transmet et que nous transmettons à autrui ; mais aussi comme process et/ou valeur cardinale sur laquelle s'établit et fonctionne (ou non) le lien social. Autrement dit, je communique à autrui - et autrui me communique - des informations sur lesquelles nous allons nous fonder pour nous accorder, ou non, notre confiance mutuelle ; et la confiance constitue, ou non, un élément capital de la matrice sociale dans laquelle nous évoluons collectivement.

Relativement à ce thème, nous nous bornerons à évoquer les trois points suivants.

D'abord, ce sujet de la confiance «en communication » et dans les organisations (au sens large) est devenu parfaitement central aujourd'hui pour les sciences humaines et sociales. Il est étudié dans l'ensemble des domaines de recherches. Toutefois, il n'est pas appréhendé de manière homogène: le nombre de variables (ou dimensions) de la confiance varie beaucoup d'un auteur à l'autre, d'une discipline à l'autre. Selon AnneMarie Gagné (2011, passim), qui travaille sur le thème « les relations publiques à l'ère de l'entreprise 'responsable' » et qui a recensé les divers modèles de la confiance issus des disciplines clefs : "en plus de constater la diversité croissante des champs d'application de la confiance (gestion, sociologie, marketing, psychologie, etc.), [l'on peut observer] une profusion de termes ou d'éléments faisant référence à la confiance : coopération, risque, incertitude, fiabilité, opportunisme, etc.». Et, pour suivre: "l'analyse de l'ensemble des modèles de confiance conduit à dégager quatre ensembles constitutifs de la confiance: la composante attitudinale (ouverture d'esprit, justice, disponibilité, honnêteté, bienveillance, équité, compétence et expertise perçues), la composante relationnelle (communication, interdépendance, continuité, fréquence, existence de routines communes), la composante organisationnelle (structures de l'entreprise, normes, valeurs, capacités techniques, légitimité) et, enfin, la composante stratégique (respect des engagements et des promesses, cohérence entre le discours et les actes) ».

Quoiqu'il en soit, et quelque soit le champ de recherche envisagé, la confiance est finalement considérée comme un facteur crucial du fonctionnement social. Ainsi, selon Alain Peyrefitte, auteur de La société de confiance (1995) et du Mal français (1976) mal qui consiste justement en grande partie en un manque flagrant de confiance, notamment de la part des entrepreneurs du pays -, c'est le comportement des gens et la confiance qu'ils s'accordent qui créent le développement, le capital et le travail n'étant que des «facteurs seconds ». Pour le sociologue Georg Simmel (in Etudes sur les formes de la socialisation, 1908), la confiance est "de toute évidence l'une des forces de synthèse les plus importantes au sein de la société » et cette dernière se désintégrerait s'il n'y avait pas une confiance généralisée entre ses membres. Pour le Prix Nobel d'Economie (1972) Kenneth Arrow, la richesse des nations ne dépendrait pas tant de facteurs économiques traditionnels tels que le travail ou l'accumulation de capital physique et humain que seulement de la confiance. Enfin, Francis Fukuyama (1996, passim), surtout connu pour sa réflexion sur «La fin de l'histoire », distingue les pays à confiance élevée (comme le Japon ou l'Allemagne), dont les économies seraient performantes, et les pays à confiance faible (au premier rang desquels la France et l'Italie), dont les résultats seraient médiocres. 
C'est ainsi que dans une contribution, intitulée Un drame - La société de défiance, à un ouvrage dédié à la confiance en entreprise - qui, d'une façon générale, considère qu'à la base de ce que nous connaissons abondamment dans les organisations aujourd'hui (stress, absentéisme, burn-out, désengagement des cadres, etc.) se trouve un déficit de confiance -, Algan, Cahuc et Zylberberg (in Institut Montaigne, 2014, passim), citant l'un des inventeurs du concept de « capital social ${ }^{28}$, James Coleman, considèrent qu' : "Un individu est confiant s'il met des ressources à disposition d'une autre partie, en l'absence d'un contrat formel, en espérant en retirer des bénéfices. La défiance mesure la peur d'être trahi dans les relations de coopération mutuellement bénéfiques ». Et ils citent eux-mêmes Arrow, selon lequel: "Virtuellement, tout échange commercial contient une part de confiance [...]. On peut vraisemblablement soutenir qu'une grande part du retard de développement économique d'une société est due à l'absence de confiance entre ses citoyens ». Autrement dit, dans nos sociétés techniquement hyper complexes, la confiance facilite les échanges, alors que la défiance agit comme un véritable frein et entrave leur développement. Algan, Cahuc et Zylberberg poursuivent : "l'intuition d'Arrow a été confirmée par une multitude de travaux qui ont mis en lumière la relation entre la confiance et une variété de phénomènes économiques, tels que l'investissement et l'innovation, le développement des échanges entre pays, le choix des actifs financiers, la taille et l'organisation des entreprises, ou encore la croissance. Dans les économies modernes, qui reposent sur les services et l'innovation, les initiatives individuelles et la coopération jouent un rôle primordial. Les capacités des entreprises à développer des méthodes de travail horizontales, qui donnent toute leur place à l'initiative et à la responsabilité de chacun, est donc essentielle pour promouvoir la croissance et le bien-être. Aujourd'hui, performance économique et sociale vont de pair ». Notons que, de ce point de vue, les français apparaissent particulièrement méfiants, comparés au reste de la planète : parmi les 97 pays (couverts par des enquêtes dignes de foi), la France se situe au $58^{\text {ème }}$ rang sur l'échelle de confiance interpersonnelle (en 2007, seulement 22\% des français déclaraient faire confiance aux autres).

Enfin, pour ouvrir la discussion et le débat, c'est in fine une interrogation que nous poserons : comment, grâce à la (ou en matière de) communication, sinon établir la confiance dans nos systèmes et nos organisations, en permettre à terme l'émergence - au sens systémique du terme, c'est-à-dire permettre ou favoriser l'apparition de nouvelles propriétés, à partir de certains seuils critiques, dans les systèmes complexes que constituent les organisations?

D'emblée, et compte-tenu de la conception de la confiance principalement proposée ici - à savoir qu'elle comporte un élément de choix, de "pari», de "saut dans l'engagement »-, nous dirons que la confiance ne peut s'ordonner: elle "ne peut être forcée ou imposée : elle est volontaire, spontanée et naturelle; elle se co-construit dans les relations bilatérales qui s'établissent entre individus ou entre groupes » (selon Maurel, Chebbi, 2012, passim). En d'autres termes, nous soulignerons ce paradoxe : on peut avoir toutes les meilleures raisons du monde d'octroyer sa confiance à autrui sans

\footnotetext{
${ }^{28}$ Dans Les formes de capital (1986) le sociologue Pierre Bourdieu différencie trois formes de capital : le capital économique, le capital culturel et le capital social ; cette dernière forme étant définie comme : «l'ensemble des ressources actuelles ou potentielles qui sont liées à la possession d'un réseau durable de relations plus ou moins institutionnalisées d'interconnaissance et d'interreconnaissance ».
} 
finalement le faire et, inversement, toutes les meilleures raisons de la refuser mais in fine l'accorder!

Pour tenter une première réponse à la question, nous affirmerons que, bien évidemment, nous ne disposons d'aucune recette... Toutefois, si l'on prend le cas de la France, Algan, Cahuc, Zylberberg (ibid.) notent que: «[en France] Notre défiance s'accompagne de la perception d'une société hiérarchique, marquée par des relations sociales distantes et conflictuelles. Interrogés sur la stratification de leur société, deux Français sur trois la considèrent organisée de façon pyramidale, avec une petite élite au sommet, une petite classe moyenne, et Ia majorité des citoyens en bas de l'échelle sociale [...] La sensation de vivre dans une société hiérarchique s'accompagne du sentiment que la concurrence est déloyale et que les règles sont systématiquement contournées. Ainsi, nous suspectons particulièrement les riches et nos élites de ne respecter aucune règle: $52 \%$ des Français estiment que de nos jours, on ne peut arriver au sommet sans être corrompu, alors que cette part n'excède pas $20 \%$ aux États-Unis, en Angleterre ou en Norvège ». En d'autres termes, "Les Français expriment bien plus souvent que les citoyens des autres pays développés le sentiment de vivre dans une société hiérarchique qui évince la réciprocité et Ia coopération [...] Notre défiance résulte d'un cercle vicieux qui nous distingue des autres pays. Le fonctionnement hiérarchique et élitiste de l'école nourrit celui des entreprises et de l'État. Plus qu'ailleurs, notre école est en charge de Ia seule transmission du savoir, au détriment de l'apprentissage de la coopération », (id., ibid.).

Dès lors, si l'on considère la communication à la fois comme transmission d'informations et matrice sociale, la notion d'habitus, chère au sociologue $\mathrm{P}$. Bourdieu (1980, passim), apparaît particulièrement éclairante relativement à la problématique. Elle peut être définie comme: "systèmes de dispositions durables et transposables [...] disposées à fonctionner [...] en tant que principes générateurs et organisateurs de pratiques et de représentations qui peuvent être objectivement adaptées à leur but sans supposer la visée consciente de fin et la maîtrise expresse des opérations nécessaires pour les atteindre ». La question se précise alors: comment modifier nos habitus de défiance? Deux pistes nous paraissent pouvoir être compendieusement synthétisées par les deux assertions suivantes :

- «Le partage d'informations constitue l'un des facteurs de confiance entre individus ou groupes dans l'organisation »;

- «Si la confiance organisationnelle n'est pas présente, il faut la construire en développant des normes, des obligations ou des valeurs partagées » (voir Maurel, Chebbi, ibid.).

Vaste programme...

\section{Références bibliographiques}

BEJEAN S., MIDY F., PEYRON C. (1999), « La rationalité simonienne : interprétations et enjeux épistémologiques », Novembre 1999, sur le lien http://leg.ubourgogne.fr/documents-de-travail/e9914.pdf

BENOIT D. (2013), Information-Communication : Théories - Pratiques - Ethique - De la psychothérapie aux techniques de vente, Editions ESKA, Paris. 
BENOIT D. (dir.), (2012), Ethique et Communication, Editions U.E., Saarbrücken (Allemagne).

BENOIT D. (2004), « Le constructivisme en communication : une évidence à revisiter », in Questions de communication, Psychologie sociale et effets des médias, $\mathrm{n}^{\circ} 4$, septembre 2004, pp. 185-202.

BERNOUX P. (2004), Sociologie $d u$ changement dans les entreprises et les organisations, Seuil, Paris.

BOBIN C. (2012), L'homme-joie, L'Iconoclaste, Paris.

BOURDIEU P. (1980), Le sens pratique, Éditions de Minuit, Paris.

BRETON P., PROULX S. (2002), L'explosion de la communication à l'aube du XXIème siècle, La Découverte, Paris.

CHAMPAGNE P. (2001), «L'arroseur arrosé (1) : le 'baromètre'sur les Français et les médias », février 2001, sur le lien : http://www.acrimed.org/article104.html

COMTE-SPONVILLE A. (2013), Dictionnaire philosophique, Paris, PUF.

COOPER L., SHEPARD R. (1985), "Le retournement mental des objets », Pour la Science $\mathrm{n}^{\circ} 88$ - février 1985, pp. 40-47.

COZIC M. (2008), «La rationalité limitée », ENS Ulm \& IHPST, ENS Ulm, Etudes Cognitives, sur le lien : http://mikael.cozic.free.fr/rationalitelimitee.pdf

DELEUZE G., GUATTARI F. (1980), Mille Plateaux, Éditions de Minuit, Paris.

DESJARDINS A. (2005), Bienvenue sur la voie, Editions de La Table Ronde, Paris.

DUPUY F. (Centre européen d'éducation permanente, Cedep, Insead) (2012), « Pour une révolution managériale : rétablir la confiance et l'engagement », in Le Monde Economie 12.11.2012.

FUKUYAMA F. (1996), Trust - The social virtues and the creation of prosperity, The Free Press, New York.

GAGNE A.-M. (2011), « La confiance et le soupçon - Faire des relations publiques à l'ère de l'entreprise 'responsable' », Communication [En ligne], Vol. 29/1 |2011, mis en ligne le 04 octobre 2011, consulté le 05 avril 2015. URL : http://communication.revues.org/2451 ; DOI : 10.4000/communication.2451

GUIBERT N. (1998), "La confiance en marketing : fondements et applications », Recherche et application en marketing, 14(1) DOI : 10.1177/076737019901400101

GURVIEZ P. (1998), «Le rôle central de la confiance dans la relation consommateurmarque », Thèse de doctorat en sciences de gestion, Aix-en-Provence, Université AixMarseille 3.

INSTITUT MONTAIGNE (2014), Et la confiance, Bordel ? Faire le pari de la confiance en entreprise, Eyrolles (voir notamment Algan Y., Cahuc P., Zylberberg A., "Un drame - La société de défiance»; Malarewicz J.-A., "les ambiguïtés du développement personnel en entreprise »).

JOLLIEN A. (2012), Petit traité de l'abandon, Seuil, Paris.

JULIEN F. (2014), "Qu'est-ce qu'un concept?», Cours de Philosophie, BNF, 12.11.2014, sur le lien https://www.youtube.com/watch?v=P116R_V312I

JYOJI T. (2001), L'Art du kôan zen, Albin Michel, Paris.

KORZYBSKI A. (1933), Science and Sanity. An introduction to non-Aristotelian systems and General Semantics, The International non-Aristotelician Library Publishing Compagny, Lakeville, Connecticut (USA). 
LAPLANE D. (1999), «Controverse : existe-t-il une pensée sans langage ?», La Recherche, mensuel $\mathrm{n}^{\circ} 325$ daté de novembre 1999, p. 62, voir le lien http://www.larecherche.fr/savoirs/autre/controverse-existe-t-il-pensee-langage-01-111999-88994

LAPLANE D. (2001), « La pensée sans langage », Études, 2001/3 (Tome 394), pp. 345 357.

LAUFER R., ORILLARD M. (2000), (dir.), La confiance en question, L'Harmattan, Paris.

LE CARDINAL G., GUYONNET J.-F., POUZOULLIC B. (1997), La dynamique de la confiance, Dunod, Paris.

LELOUP J.-Y. (2014), De Nietzsche à Maître Eckhart, Almora, Paris.

MASSIN C. (2016), Une vie en confiance : dialogues sur la peur et autres folies, Odile Jacob, Paris.

MARZANO M. (2011), "Qu'est ce que la Confiance ? », Etudes, janvier 2010, tome 412/1, La Tribune, "Une société où la confiance n'existe plus perd ses bases », avril 2011.

MAUREL D., CHEBBI A. (2012), « La perception de la confiance informationnelle », Communication et Organisation [En ligne], 42 |2012, mis en ligne le 01 décembre 2014, consulté le 22 juillet 2013. URL : http://communicationorganisation.revues.org/3864

PEYREFITTE A. (1995), « La société de confiance - Essai sur les origines et la nature du développement $»$, Odile Jacob, Paris.

QUERE L. (2001), « La structure cognitive et normative de la confiance », Réseaux (La Découverte), 2001/4, n 108, pp. 125 - 152.

RAMANANTSOA B., REITTER R. (2012), Confiance et défiance dans les organisations, Economica.

SIMON H.A. (1976), De la rationalité substantive à la rationalité procédurale, sur le lien http://www.mcxapc.org/fileadmin/docs/lesintrouvables/simon5.pdf

SIREIX L., DUBOIS J.-L. (1999), «Vers un modèle qualité-satisfaction intégrant la confiance $»$, Recherche et application en marketing 14(3), pp.1-22 - DOI : 10.1177/076737019901400301

TEYSSIER J.-P. (2004), Frapper sans heurter - Quelle éthique pour la publicité ? Armand Colin, Paris.

THUDEROZ C., MANGEMATIN V., HARRISON D. (1999), La confiance. Approches économiques et sociologiques, Gaëtan Morin, Montréal.

WATZLAWICK P. (1978), La réalité de la réalité, Seuil, Paris.

WEBER M. (1959), Le savant et le politique, Plon, Paris.

WINKIN Y. (1996), Anthropologie de la communication - De la théorie au terrain, De Boeck Université, Bruxelles. 